\title{
METTL21B is a prognostic biomarker and potential therapeutic target in low-grade gliomas
}

\author{
Xin Shu ${ }^{1}$, Xinquan $\mathrm{Li}^{1}$, Xiaochen Xiang ${ }^{1}$, Qiang Wang ${ }^{1}$, Qingming $\mathrm{Wu}^{1, \&}$ \\ ${ }^{1}$ Institute of Infection, Immunology and Tumor Microenvironment, Hubei Province Key Laboratory of Occupational \\ Hazard Identification and Control, Medical College, Wuhan University of Science and Technology, Wuhan 430065, \\ China
}

Correspondence to: Qiang Wang, Qingming Wu; email: wangqiang@wust.edu.cn; wuhe9224@sina.com, https://orcid.org/ 0000-0003-4484-1090

Keywords: eukaryotic translation elongation factor $1 \mathrm{~A}(\mathrm{eEF} 1 \mathrm{~A})$, methyltransferase, METTL21B, low grade glioma, prognosis Received: April 9, $2021 \quad$ Accepted: August 14, $2021 \quad$ Published: August 26, 2021

Copyright: (C) 2021 Shu et al. This is an open access article distributed under the terms of the Creative Commons Attribution License (CC BY 3.0), which permits unrestricted use, distribution, and reproduction in any medium, provided the original author and source are credited.

\section{ABSTRACT}

A considerable amount of literature has demonstrated that eukaryotic translation elongation factor 1A (eEF1A) is closely related to tumors. As a newly identified lysine specific methyltransferase targeting eEF1A at Lys-165, too little attention has been paid to the function of METTL21B. To determine the potential significance and prognostic value of METTL21B in low grade glioma (LGG), we analyzed the expression, methylation level and copy number variations (CNV) of METTL21B and its effect on prognosis in patients with LGG by 4 public databases in conjunction with experimental examination of LGG patient samples. As a result, we found that high expression, hypomethylation and gain/amplification of CNV of METTL21B were associated with poor prognosis in LGG. The potential functions of METTL21B in LGG may be involved in cell adhesion, angiogenesis and cell proliferation of tumor by enrichment analysis. In addition, METTL21B may facilitate immune evasion of tumor and affect prognosis by mediating macrophage polarization from $\mathrm{M} 1$ to $\mathrm{M} 2$ and regulating expression of immune checkpoints. Nevertheless, patients with high METTL21B level are likely to have better response to immune checkpoints blockage therapy. Because of its substrate specificity, METTL21B is expected to be a promising target for the treatment of glioma.

\section{INTRODUCTION}

Arising from glial cells, glioma is a type of primary neoplasms of center nervous system (CNS) system in adults, with the highest morbidity and mortality [1]. According to 2016 World Health Organization (WHO) classification standard of tumors of CNS, gliomas are divided into four grades (I, II, III, and IV), among which grades I, II and III are attributed as low-grade gliomas (LGGs) [2]. Although the patients with LGG had a longer survival time compared with glioblastoma (GBM, WHO grade IV), its high postoperative recurrence and mortality rates are still significant concerns [3]. More than half of LGG patients died of recurrence and progression [4].
Despite the developments of treatment for LGG in recent years, some tumors still showed therapeutic resistance ultimately and even progressed to GBM $[5,6]$. Genetic heterogeneity of LGG is considered as the main reason of different treatment responses and widely variable prognosis $[3,7]$. For example, a great quantity of studies demonstrated that IDH mutation increases the sensitivity of temozolomide chemotherapy and represents a favorable prognostic factor in LGG patients $[8,9]$. In addition, some recent research also reported that MGMT promoter methylation, TERT promoter mutation, TLX1NB, ARL9 and $1 \mathrm{p} / 19 \mathrm{q}$ deletion are association with prognosis of patients with LGG [10-14]. Taken together, identification of molecular biomarkers is 
unquestionably essential and urgent for accurately predicting prognosis and for seeking critical therapeutic targets in LGG.

Protein methylation is ubiquitous and acts as a pivotal regulator of various cellular biological processes in human and other organisms [15, 16]. Increasingly emerging evidence has found that histone and nonhistone methylation are involved in cellular pathways associated with cancer [17-19]. METTL21B, also known as FAM119B or EEF1AKMT3, is a member of methyltransferase-like protein family which contain a seven-beta-strand methyltransferase domain and can regulate methylation modification of a variety of substrates including proteins and nucleic acids [20]. Dysregulation of METTL21B has been implicated in several human diseases and pathological processes, such as multiple sclerosis, rheumatoid arthritis and neurodegenerative disease [20-22]. Eukaryotic translation elongation factor $1 \mathrm{~A}(\mathrm{eEF} 1 \mathrm{~A})$, as a subunit of eEF1 which promotes the delivery of aminoacyltRNA to the ribosome during translation, has been demonstrated as the only target substrate of METTL21B to the present date [23, 24]. Several recent studies have showed that METTL21B can catalyze the methylation of eEF1A on Lys-165 and impose a significant impact on translation of mRNAs [23, 24]. There is a tight relationship between dysregulation of eEF1A and cancer. For instance, overexpression of eEF1 is associated with poor prognosis of many types of cancers including breast, lung and liver cancer [25]. Besides, di-methylation of eEF1A lysine 55 (eEF1AK55me2) catalyzed by METTL13 can increase translational output and then promote tumorigenesis [26]. However, the prognostic significance and molecular function of METTL21B is still undetermined in glioma.

In our research, by using The Cancer Genome Atlas (TCGA), the Chinese Glioma Genome Atlas (CGGA), Rembrandt dataset and Gravendeel/GSE16011 dataset, we assessed the expression level (in conjunction with experimental examination of LGG patient samples), methylation level and copy-number variations of METTL21B in LGG tissues, analyzed the association between prognosis and expression of METTL21B, and explored the underlying molecular mechanisms of METTL21B in LGG. Furthermore, to increase the understanding of function of METTL21B in tumor immune microenvironment, we evaluated the effect of METTL21B on immune infiltration level and expression of immune checkpoints in LGG. Additionally, we also analyzed the correlation between METTL21B and four other genes which were also involved in the methylation regulation of eEF1A, and established the prognostic nomogram.

\section{MATERIALS AND METHODS}

\section{Data mining}

4 glioma public datasets comprising 1253 LGG patients (TCGA-LGG cohort: 509 patients; CGGA database: 443 patients; Rembrandt dataset: 184 patients and Gravendeel/GSE16011 dataset: 117 patients) were used for analysis [6, 27-29]. Firstly, the mRNA expression levels of METTL21B were compared between LGG tissues and normal samples by using the online website Gene Expression Profiling Interactive Analysis (GEPIA) (http://gepia.cancer-pku.cn/index.html), which included expression data from TCGA and GTEx database [30]. Besides, we downloaded transcriptome data of 337 samples from GSE16011 dataset (117 tumors and 8 normal tissues) and Rembrandt dataset (184 tumors and 28 normal tissues) to verify the expression differences of METTL21B. Then, the clinical information and transcriptional profiles were extracted from TCGA-LGG cohort and CGGA database for further analysis. The methylation level and copy-number variations (CNV) data of METTL21B were also downloaded via the Cbioportal Website. (https://www.cbioportal.org/) [31].

\section{Experimental examination of LGG patient samples}

Between June 2011 and November 2014, a series of 31 low grade glioma samples were collected from patients who underwent surgery at the Department of Neurosurgery, Associated Hospital affiliated to Wuhan University of Science and Technology. Of them, 3 samples were WHO grade I (pilocytic astrocytoma), 13 cases were WHO grade II and 15 cases were WHO grade III. All patients were followed up after hospitalization with a mean duration of 75 months (range 36-122 months). The follow-up period was calculated as the duration from the date of surgery to death, or until April 2021 for surviving patients. Recurrence was defined as local tumor growth on the basis of clinical symptoms and neuroimaging findings. This study was approved by local ethical authorities of Medical school of Wuhan University of Science and Technology in accordance with the Helsinki Criteria (No. 202176). Written informed consent was obtained from each individual patient.

Briefly, samples were dissected into two halves with one half immediately frozen at $-80^{\circ} \mathrm{C}$ for RNA extraction and the second half fixed with $10 \%$ formalin and embedded in paraffin for immunohistochemistry (IHC) staining. RNA isolation and qPCR were performed as described previously [32]. The primer sequences of human METTL21B and GAPDH were designed by GenePharma (Shanghai, China) 
(Supplementary Table 1). For IHC staining, Tissue sections were deparaffinized in xylene, rehydrated through an ethanol gradient and washed for $15 \mathrm{~min}$ with phosphate-buffered saline. For antigen retrieval, sections were heated in $0.01 \mathrm{M}$ citrate buffer ( $\mathrm{pH}$ 6.0) for $20 \mathrm{~min}$ in a microwave. Endogenous peroxidase activity was blocked by $3 \%$ hydrogen peroxidase in methanol for $10 \mathrm{~min}$. Nonspecific antibody binding was blocked by incubation with the appropriate serum for $20 \mathrm{~min}$ which reduced positive signals in vascular, stromal and blood cells. Sections were incubated with primary antibody (HPA043020, 1:500, Sigma-Aldrich Co. Ltd, German) overnight at $4^{\circ} \mathrm{C}$ in a humidified chamber and were subsequently treated with biotinylated secondary antibodies against mouse IgGs for 30 min (ABC Elite, Vector Laboratories, Burlingame, $\mathrm{CA})$ and for $30 \mathrm{~min}$ with avidin-biotin complex (ABC Elite), followed by treatment with $0.06 \%$ diaminobenzidine (Sigma Chemical, St. Louis, MO, USA) and $0.01 \%$ hydrogen peroxidase for $5 \mathrm{~min}$. All sections were counterstained with hematoxylin. Antibody specificity control stains were prepared by omitting primary antibodies. The percentage of cells stained positive compared to the total number of tumor cells was determined using Image $\mathbf{J}$ software $(\mathrm{NIH}$, Bethesda, MD, USA) by counting around 400 tumor cells at 400X magnification.

\section{Prognosis analysis}

According to the expression values of METTL21B, patients from our clinical department and those from the employed database were independently split into two groups (low vs. high expression group) based on the median value. Survival analysis was performed by Kaplan-Meier method to analyze the effect of expression, methylation and CNV of METTL21B on prognosis of LGG patients. The timeROC curve was plotted to evaluate the accuracy of METTL21B for predicting prognosis of patients with LGG by timeROC package of $\mathrm{R}$ software. In addition, multivariable cox regression analysis was employed to adjust for clinicopathological features including age, gender, tumor grade, IDH mutation status and radiotherapy.

\section{DEGs, KEGG pathways, GO analysis and gene sets enrichment analysis (GSEA)}

In TCGA-LGG database, differential expression genes (DEGs) between low and high METTL21B expression group were identified by Limma package of $\mathrm{R}$ software. $\log _{2} \mid$ fold change $\mid>1$ and adjusted $P<0.05$ were defined as the thresholds for DEGs screening. Functional enrichment analyses including Gene Ontology (GO) and Kyoto Encyclopedia of Genes and Genomes (KEGG) enrichment analysis of DEGs were performed to better understand the underlying function and carcinogenesis of METTL21B in LGG. KEGG pathway analysis was carried out by ClusterProfiler package of $\mathrm{R}$ software, and GO enrichment was analyzed by Metascape database. In additional, we utilized the MSigDb Hallmark gene set to execute the gene sets enrichment analysis by GSEA software (v4.1.0).

\section{Analysis of correlation between METTL21B and immune signature}

Tumor Immune Estimation Resource (TIMER) (https://cistrome.shinyapps.io/timer/) is a web server which can be used to evaluate levels of immune infiltration and to analyze the association between immune infiltration and prognosis [33]. The ESTIMATE immune score of each sample was calculated by applying Estimate package of $\mathrm{R}$ software. Furthermore, the CIBERSORT algorithm was used to assess composition of 22 immune cells in tumor microenvironment by CIBERSORT package.

SIGLEC15, TIGIT, PDCD1LG2, PDCD1, CD274, LAG3, HAVCR2 and CTLA4 were selected to be immune checkpoint genes [34, 35], and the association between expression levels of these genes and METTL21B was analyzed in LGG. Tumor mutation burden (TMB) was computed by Maftools R package.

\section{Construction of prognostic model of eEF1A associated methyltransferases}

Except for METTL21B, EEF1AKMT1(N6AMT2), EEF1AKMT2(METTL10), EEF1AKNMT(METTL13) and EEF1AKMT4(ECE2) are also methyltransferases involved in methylation regulation of eEF1A [36]. The association between expression of these genes and METTL21B was analyzed, and nomogram was constructed by 'rms' R package.

\section{Statistical analysis}

Survival analysis and multivariate cox analysis were performed by "survival" and "survminer" package. Quantitative variables were compared by Wilcox-test, Student's t-test or ANOVA with TukeyHSD test. Correlation analyses were assessed by using the Spearman or Pearson's correlation test. R software (v4.0.2) was used for statistical analysis and graphing. $\mathrm{P}$ $<0.05$ indicates statistical significance of difference.

\section{Availability of data and materials}

The datasets used and/or analyzed during the current study are available from the corresponding author on reasonable request. 


\section{RESULTS}

\section{Expression level of METTL21B in LGG}

We determined the difference in expression of METTL21B between tumor tissues and normal tissues for 33 types of human cancers from TCGA and GTEx database by the GEPIA website (Supplementary Figure 1A). The data indicated that mRNA expression of METTL21B in LGG was significantly up-regulated compared with normal tissues (Figure 1A). We further verified that METTL21B is highly expressed in LGG tissues by Rembrandt database (Figure 1B) and GSE16011 dataset (Figure 1C).
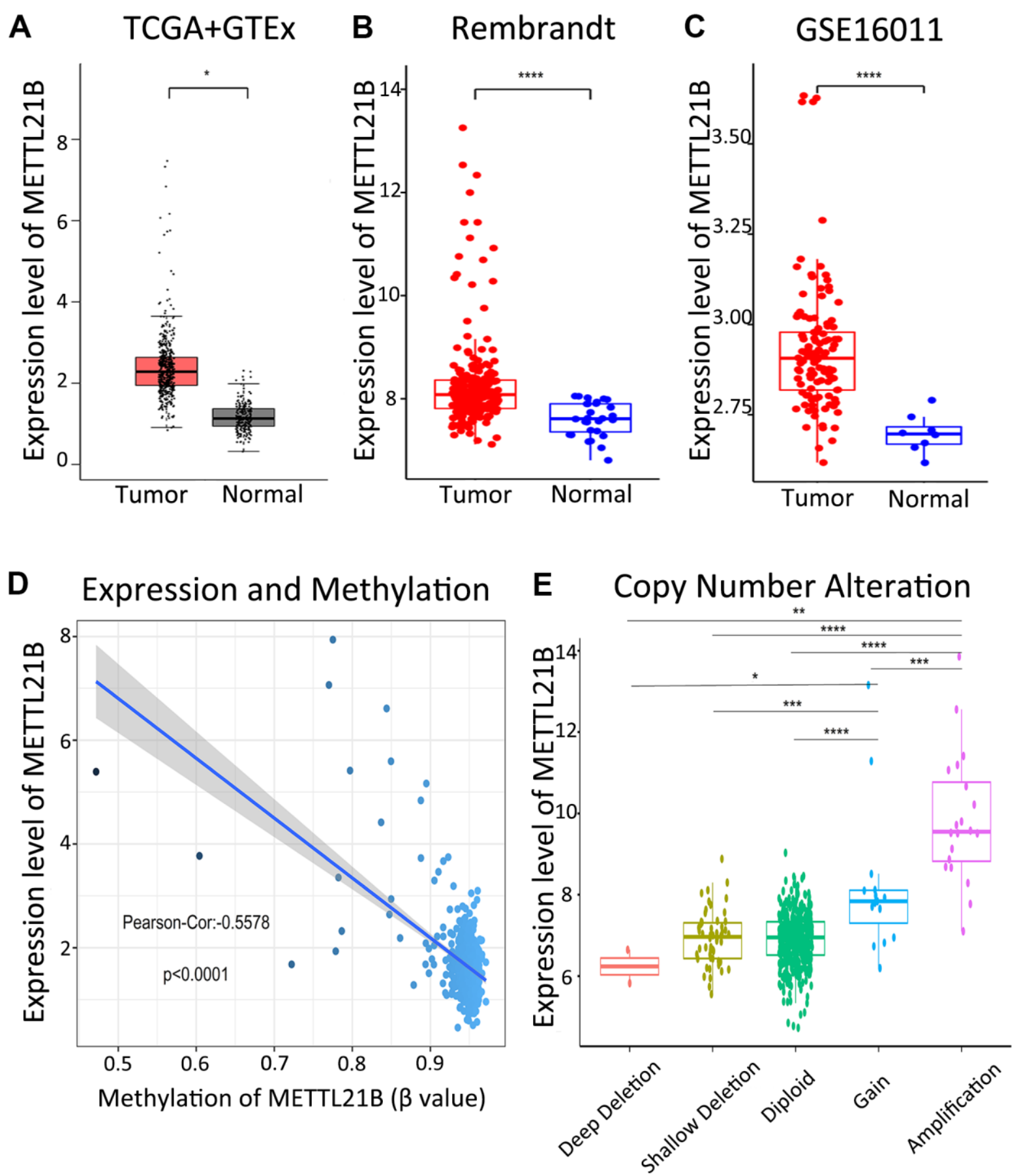

Figure 1. Expression of METTL21B in LGG. The expression differences of METTL21B between LGG tissues and normal samples in TCGA+GTEx (A), Rembrandt (B) and GSE16011 (C) dataset; (D) The correlation between expression and methylation of METTL21B. (E) Copy number gain/amplification of METTL21B markedly increased the mRNA expression. ${ }^{*} \mathrm{P}<0.05 ;{ }^{*} \mathrm{P}<0.01 ;{ }^{* * *} \mathrm{P}<0.001 ;{ }^{* * * *} \mathrm{P}<0.0001$. 
As shown in Figure 1D, a significantly negative correlation (Pearson-Cor $=-0.5578, \mathrm{P}<0.0001$ ) was observed between mRNA expression level and DNA methylation of METTL21B. Besides, we explored the effect of copy number variation on METTL21B expression and showed that the copy number gain/amplification of METTL21B markedly increased the mRNA expression, while deletion of METTL21B copy number had no effect on its mRNA expression. (Figure 1E).

By analyzing the association between METTL21B expression and clinical features including age, gender, race and WHO grade, we found that WHO grade III glioma has higher expression level of METTL21B compared with WHO grade II (Figure 2), which
A

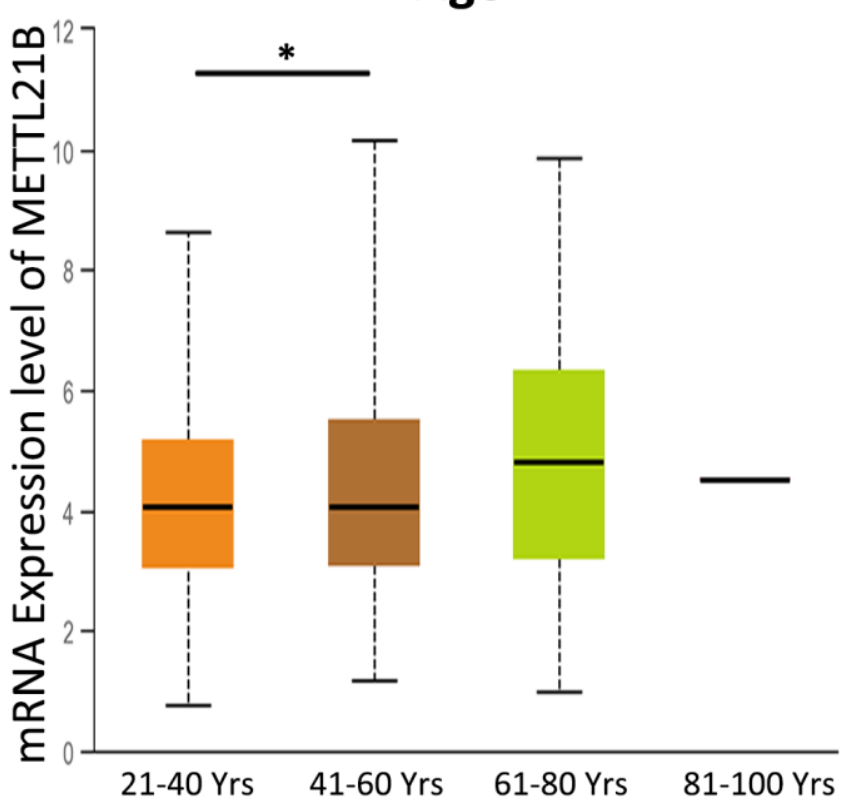

C

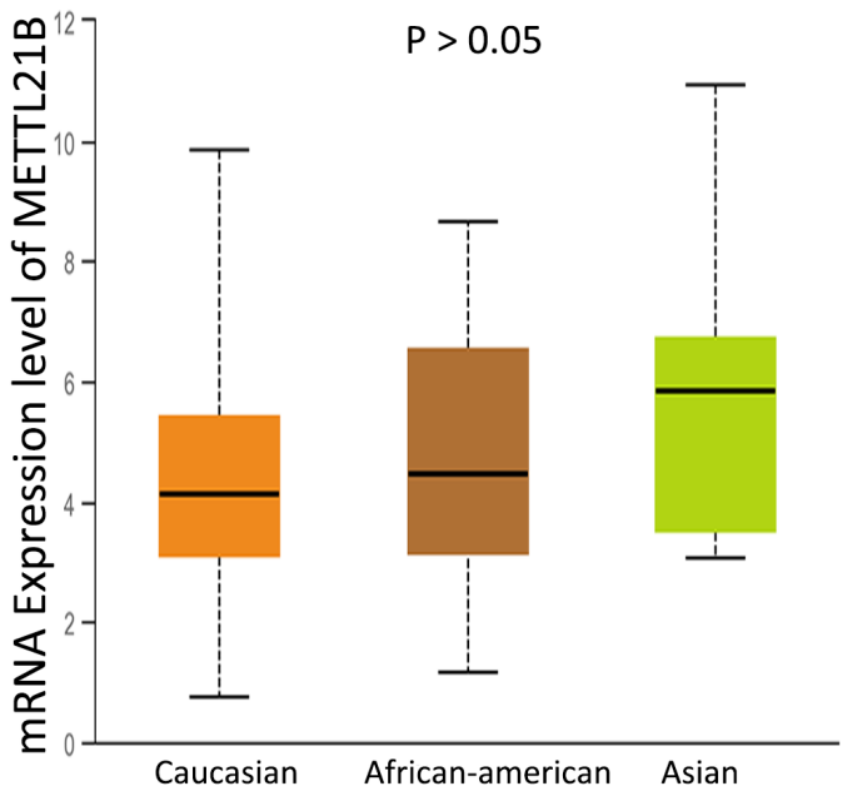

B

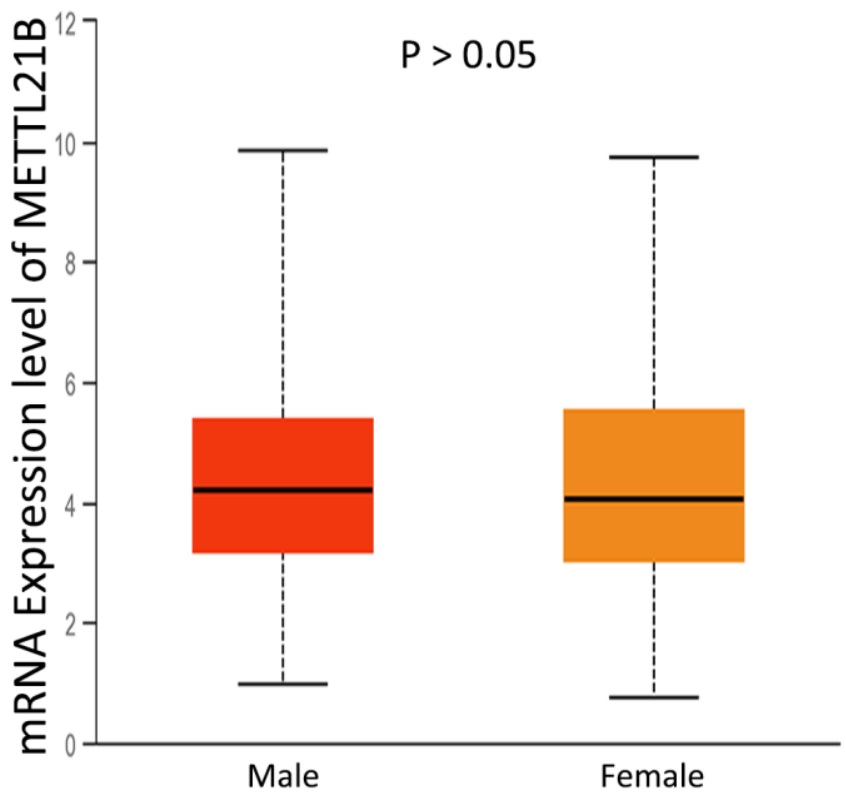

D WHO Grade

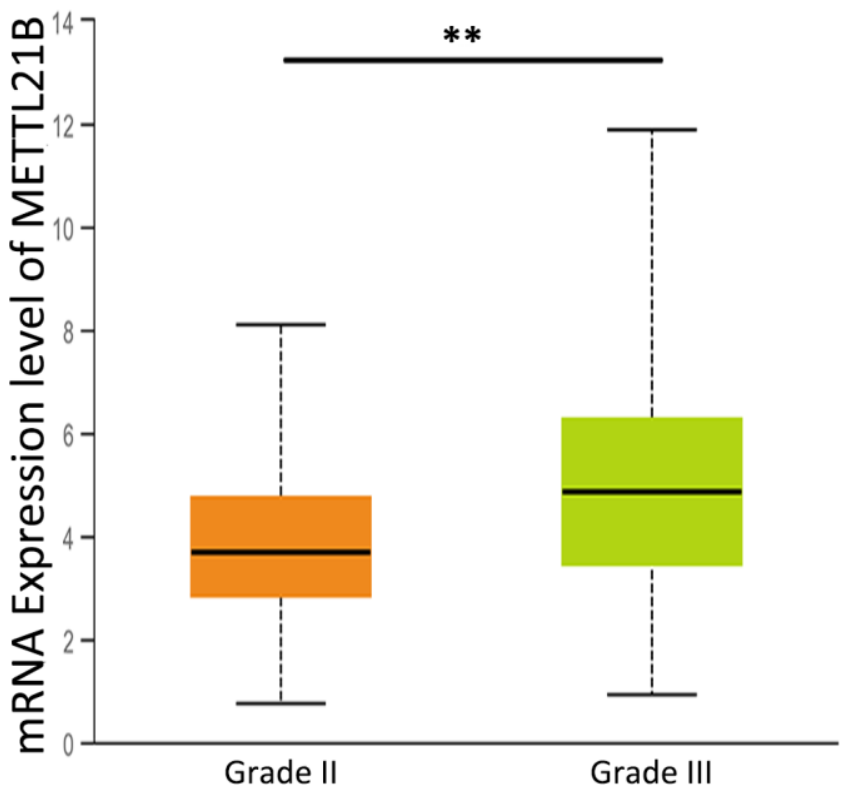

Figure 2. The association between METTL21B expression and clinical features. (A) age; (B) gender; (C) race; (D) WHO grade. ${ }^{*} \mathrm{P}<0.05 ;{ }^{*} \mathrm{P}<0.01$. 
indicates that METTL21B could be related to malignant behaviors of LGG.

We further verified the expression of METTL21B in LGG by examining a series of 31 clinical specimens. As a result, there was a significantly higher mRNA expression level in WHO grade III than in WHO grade I/II (Figure 3A). In addition, immunohistochemistry (IHC) staining confirmed that the expression of METTL21B was positively associated with WHO grade
A

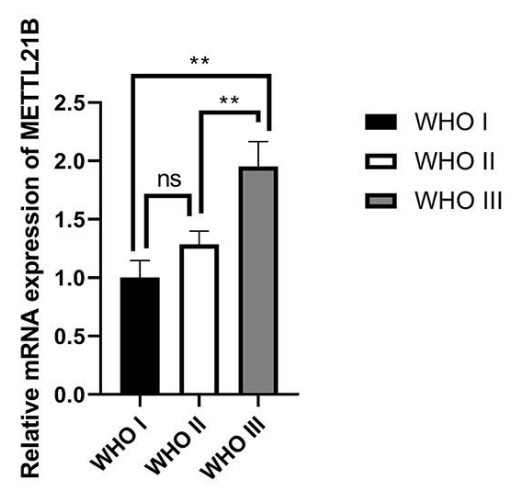

C

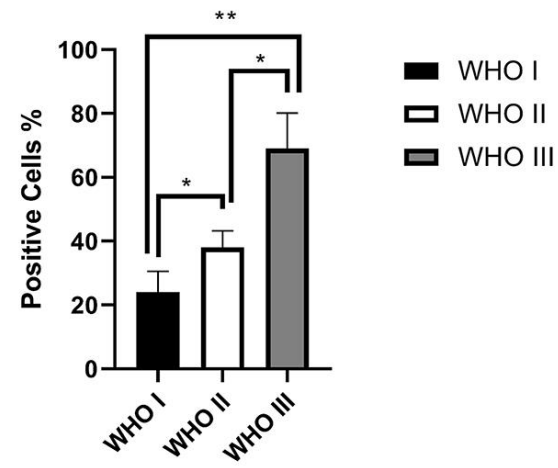

D
B
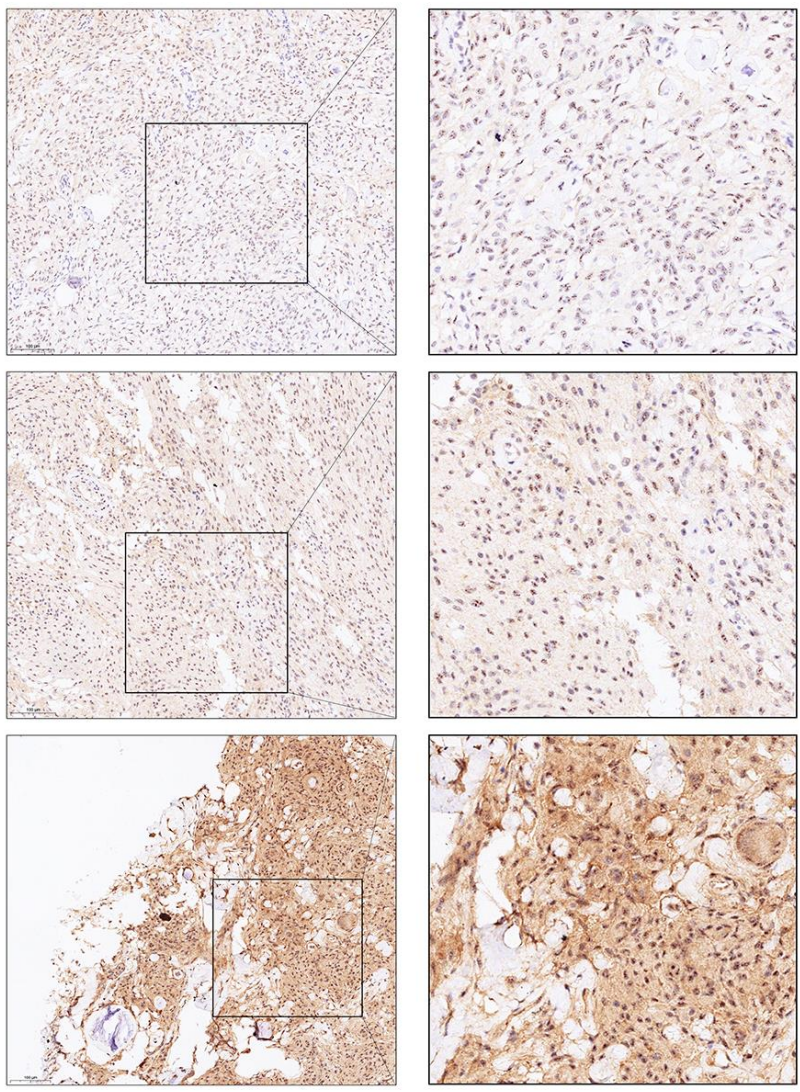

$\mathbf{E}$
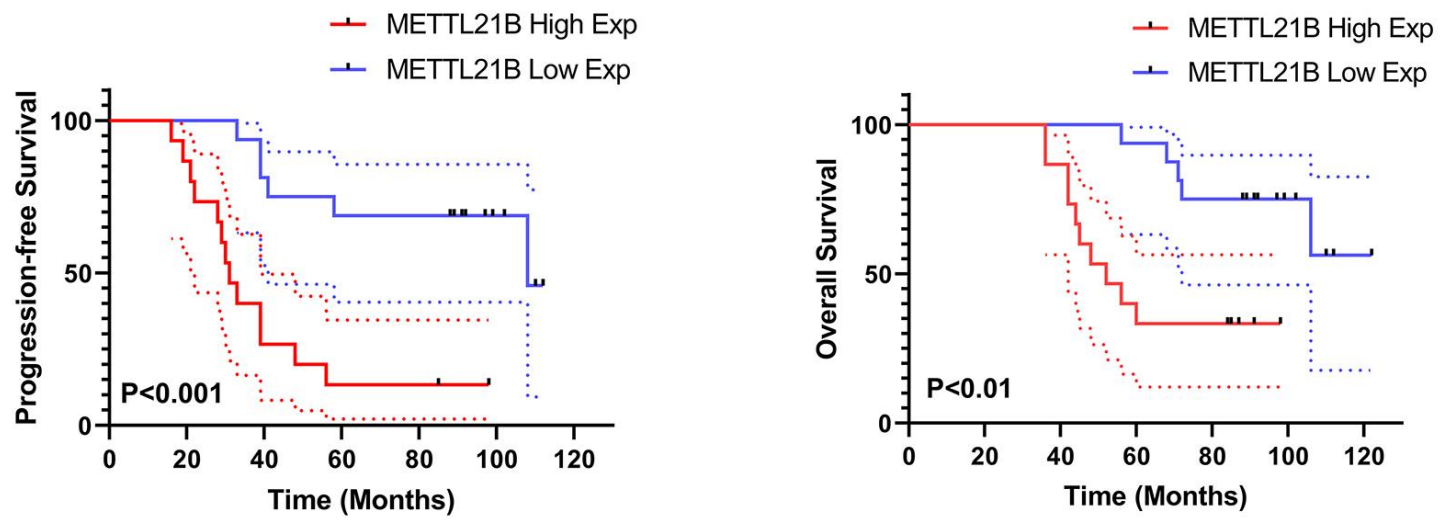

Figure 3. Experimental examination of METTL21B expression and prognosis analysis in LGG patient clinical samples. (A) The relative mRNA expression level in LGG with different WHO grades. (B) Representative immunohistochemistry images for METTL21B in LGG with different WHO grades. Positive cells showed nuclear and/or cytoplasmic brown staining while negative cells showed blue nuclei counter staining. (C) METTL21B positive cells were counted in LGG with different WHO grades. ${ }^{*} P<0.05$; ${ }^{*} P<0.01$; ns: no significance. (D, E) METTL21B high expression predicts a worse progression-free and overall survival $(\mathrm{P}<0.001$ and $\mathrm{P}<0.01$, respectively). 
at the protein level (Figure 3B, 3C). These results were in accordance with those from databases.

\section{Prognostic value of METTL21B in LGG}

Survival heat map in Supplementary Figure 1B showed the correlation of METTL21B expression and prognosis

A

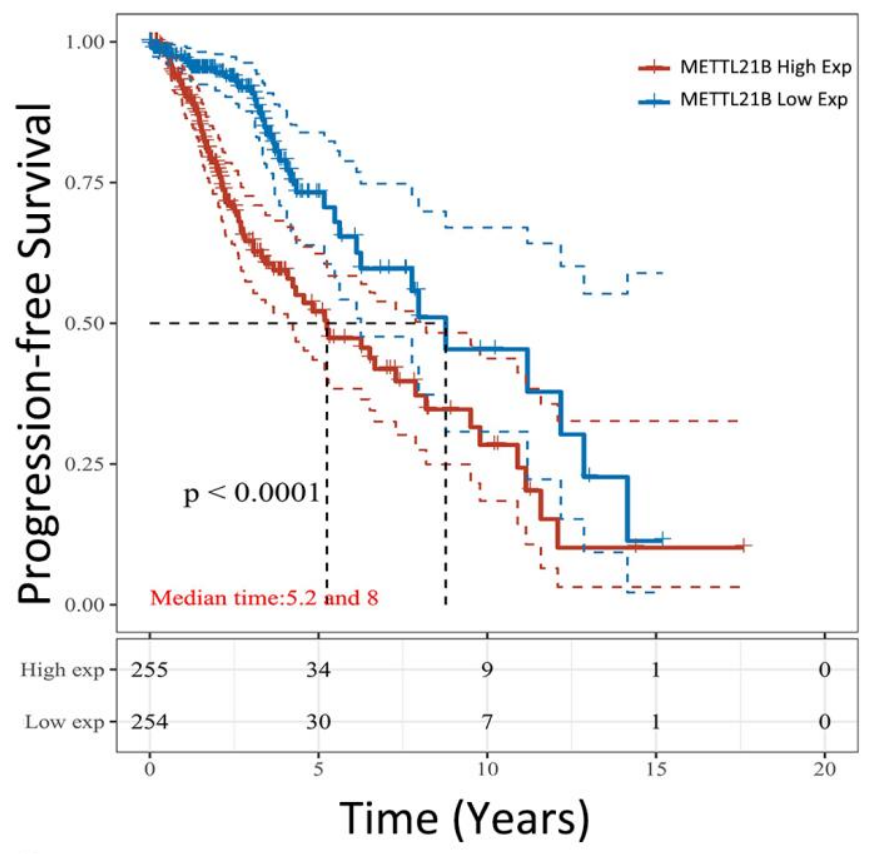

C

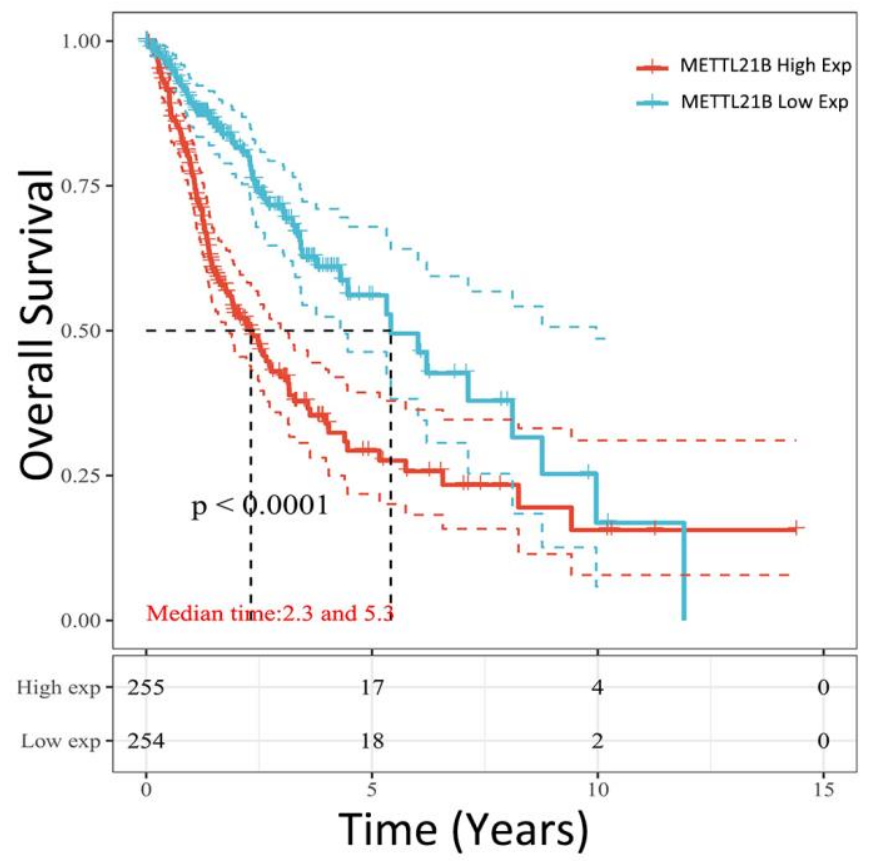

of 33 cancer types in TCGA. Although the expression of METTL21B was remarkably up-regulated or downregulated in 7 types of cancers compared with normal tissues as described in Supplementary Figure 1A, the prognosis was only associated with METTL21B expression in LGG. As exhibited in Figures 3D, 3E, 4, Kaplan-Meier curves were plotted to evaluate the effect

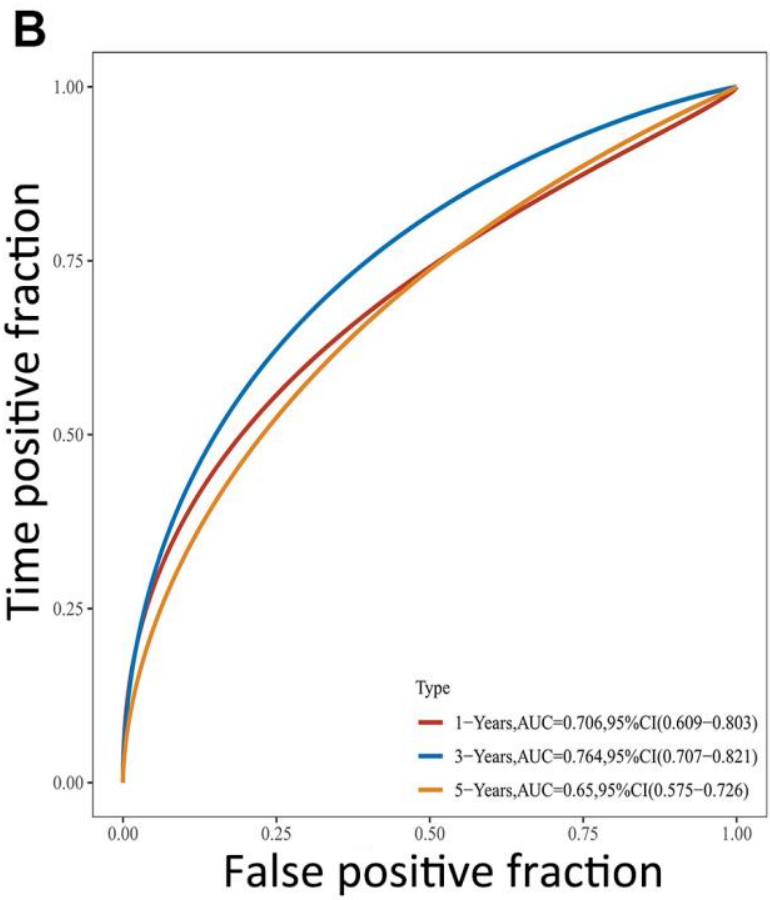

D

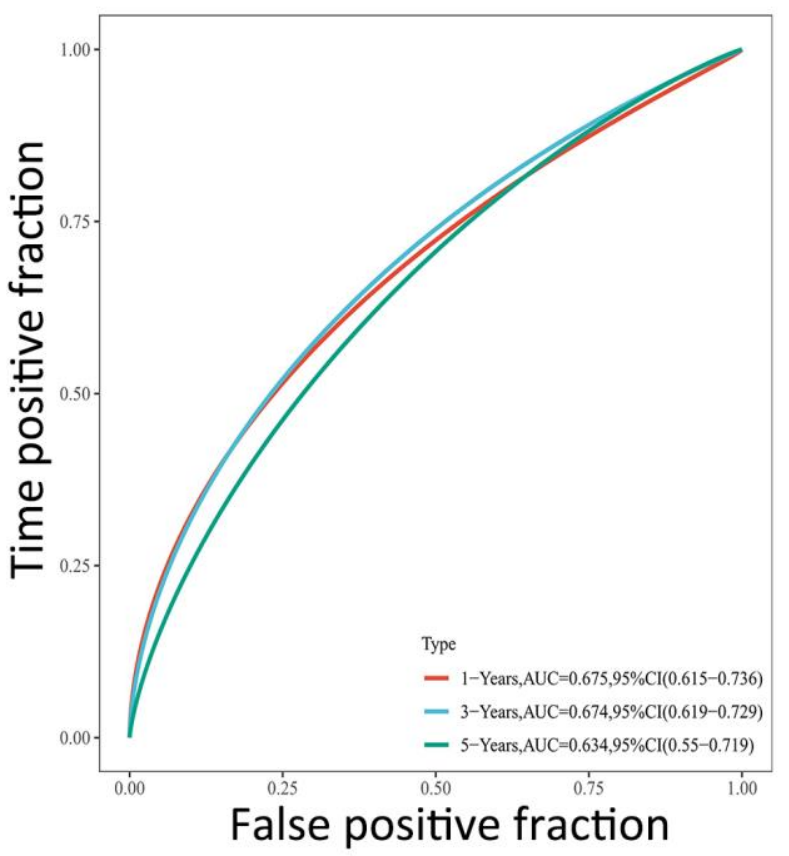

Figure 4. Prognostic value of METTL21B for patients with LGG in TCGA. The effect of METTL21B expression on overall survival (A) and progression-free survival (C) of patients; The ROC curve for OS (B) and PFS (D). 
of METTL21B on overall survival (OS) and progression-free survival (PFS) of patients in our clinical specimens and TCGA-LGG cohort. Patients with higher METTL21B expression had significantly poorer overall survival (median OS: 5.2 vs. 8.0 years, log-rank $\mathrm{p}<0.0001$ ) and progression-free survival (median PFS: 2.3 vs. 5.3 months, log-rank p<0.0001) (Figure 4A, 4C). The areas under the time-dependent ROC curves (AUC) for 1-year, 3-year and 5-year overall survival rates were $0.706,0.764$ and 0.65 respectively, which suggested METTL21B had favorable prognostic accuracy in LGG patients (Figure 4B). Besides, the poor prognosis in the group with high METTL21B expression was also validated by data from CGGA database (Supplementary Figure 2A) and Rembrandt database (Supplementary Figure 2B).

As expected, low methylation level of METTL21B was associated with poor prognosis (median OS: 5.6 vs. 9.8 years) among patients with LGG (Supplementary Figure 3A). In addition, median overall survival was shorter for patients with gain/amplification of METTL21B copy number (median OS: 2.5 vs. 7.9 years) (Supplementary Figure 3B).

Multivariate Cox proportional-hazards model analysis revealed that expression of METTL21B was still significantly correlated with $\mathrm{OS}(\mathrm{HR}=2.34, \mathrm{P}<0.0001)$ after adjusting age, gender, WHO grade, IDHmutation status and radiotherapy, demonstrating that the gene is an independent prognostic factor in LGG (Table 1).

\section{Identification of DEGs between low and high METTL21B group and KEGG and GO analysis}

To understand the biological function of METTL21B in LGG, differential expression genes (DEGs) analysis was run between low and high METTL21B expression group by Limma package of $\mathrm{R}$ software. As depicted by the heatmap and volcano plot (Supplementary Figure 4A, 4B), 71 up-regulated genes (red dots) and 185 down-regulated genes (blue dots) were identified in high METTL21B expression group. KEGG enrichment analysis of DEGs showed that these up-regulated genes are mainly involved in Human T-cell leukemia virus 1 infection, EpsteinBarr virus infection, AGE-RAGE signaling pathway, ECM-receptor interaction and focal adhesion (Figure 5A, 5B). Additionally, the chord chart displayed 5 most enriched GO terms by different colors in high METTL21B expression group, including extracellular matrix organization, regulation of cell adhesion, interferon-gamma-mediated signal, vasculature development and cell cycle (Figure 5C, 5D). The results imply that METTL21B may be associated with cell adhesion, tumor immune, angiogenesis and cell proliferation of low-grade glioma.

\section{Results of gene set enrichment analysis}

We performed single gene GSEA to explore the METTL21B-related pathways in LGG. 27 out of 50 hallmark gene sets were significantly enriched in high METTL21B expression group at nominal $p<0.01$ and FDR $<0.25$, and of which 14 gene sets with normalized enrichment score (NES) > 2.0 were listed in Table 2 . Among these gene sets, 2 gene sets are targets of E2F and MYC transcription factor families. "G2M CHECKPOINT", "EPITHELIAL_MESENCHYMAL TRANSITION", "ANGIOGENESIS", "DNA_REPAIR" and "APOPTOSIS" are involved in tumorigenesis and tumor metastasis. Besides, 6 immune-related gene sets, including "INTERFERON_GAMMA RESPONSE", "IL6_JAK_STAT3_SIGNALING", "TNFA_SIGNALING_VIA_NFKB", "INTERFERON ALPHA_RESPONSE", "IL2_STAT5_SIGNALING" and "INFLAMMATORY_RESPONSE", were also clearly up-regulated in the group with high METTL21B expression (Figure 6).

\section{Association between METTL21B and immune infiltration}

Due to the enrichment in several immune-associated signaling pathways in high METTL21B group, we investigated the association between METTL21B and immune microenvironment. The correlation between expression of METTL21B and infiltration levels of CD4+ T cells, CD8+ T cells, B cells, neutrophils, macrophage and dendritic cells was examined by TIMER website. As displayed in Figure 7A, infiltration levels of all six immune cell types were significantly positively correlated with expression of METTL21B in LGG after adjusting for tumor purity. Analogously, higher immune scores which were calculated by ESTIMATE algorithm based on transcription profile were observed in the group with up-regulated METTL21B (Figure 7B). Besides, macrophages displayed the strongest positive correlation with METTL21B expression level $(\mathrm{r}=0.415, \mathrm{p}<0.001)$, and its high level of infiltration portended unfavorable prognosis for LGG patients (Figure 7C).

We further evaluated composition of 22 subtypes of immune cells in all TCGA-LGG samples by CIBERSORT method (Figure 7D). Higher percentage of M2 macrophage and resting memory CD4+ T cell were noticed in high METTL21B group, while 
Table 1. Multivariate Cox model for infiltration of clinical features and METTL21B expression in LGG.

\begin{tabular}{lcccccc}
\hline \multirow{2}{*}{ Clinical characteristics } & \multicolumn{2}{c}{ Progression-free survival } & & \multicolumn{2}{c}{ Overall survival } \\
\cline { 2 - 3 } \cline { 5 - 6 } & HR $(\mathbf{9 5 \%} \% \mathbf{C I})$ & P value & & HR (95\%CI) & P value \\
\hline Age & $1.02(1.01-1.04)$ & $0.0003^{*}$ & & $1.05(1.03-1.07)$ & $<0.0001^{*}$ \\
Gender & $0.72(0.52-0.99)$ & $0.0444^{*}$ & & $0.96(0.63-1.46)$ & 0.8478 \\
WHO grade & $1.39(00.97-1.99)$ & 0.0721 & & $2.44(1.50-3.96)$ & $0.0003^{*}$ \\
IDH-mutation & $1.00(0.69-1.46)$ & 0.9898 & & $0.90(0.53-1.53)$ & 0.6919 \\
Radiation therapy & $0.84(0.58-1.23)$ & 0.3754 & & $1.25(0.71-2.20)$ & 0.4378 \\
METTL21B expression & $2.34(1.67-3.30)$ & $<0.0001^{*}$ & & $2.01(1.26-3.20)$ & $0.0034^{*}$ \\
\hline
\end{tabular}

infiltration levels of $\mathrm{B}$ cell plasma and $\mathrm{T}$ cell follicular helper were low (Figure 7E).

\section{METTL21B is associated with immune checkpoints and tumor mutation burden}

Increased expression of 7 immune checkpoints (CD274, CTLA4, HAVCR2, LAG3, PDCD1, PDCD1LG2 and SIGLEC15) were found in high METTL21B expression group (Supplementary Figure 5). Similarly, a significant positive correlation between expression of these immune checkpoints and METTL21B was confirmed by Spearman correlation analysis (Figure 8A). Of note, overexpression of all these 7 genes were associated with low OS in patients with LGG (Figure 8B-8H), which indicated METTL21B could be involved tumor immune escape and promote progression by regulating expression of immune checkpoints. Besides, the tumor mutation burden of LGG was markedly positively correlated with METTL21B, implying that patients with high expression level of METTL21B may be more likely to have favorable response to immunotherapy targeting immune checkpoints (Figure 8I).

\section{Construction of prognostic model of METTL21B along with other eEF1A associated methyltransferases}

EEF1AKMT1 (N6AMT2), EEF1AKMT2 (METTL10), EEF1AKNMT (METTL13) and EEF1AKMT4 (ECE2) are also regulatory factors of methylation modification of eEF1A. Taking this into consideration, we established a prognostic model of METTL21B along with other eEF1A associated methyltransferases in LGG.-Multivariate Cox regression analysis found that age, WHO grade, METTL21B and EEF1AKMT2 were independent prognostic factors in LGG (Figure 9A).

Next, nomogram was plotted to visualize the model, by which the total points can be obtained to evaluate survival possibility of each patient (Figure 9B). C-index of the model is 0.81 , which indicated the excellent predictive ability.

\section{DISCUSSION}

As a newly identified lysine specific methyltransferase targeting eEF1 A at Lys-165, too little attention has been paid to the function of METTL21B and the precise role of METTL21B in LGG are still unclear. In this study, we provide the first insights into the clinical significance and biological function of METTL21B in LGG by performing a series of in-depth bioinformatics analysis in conjunction with experimental examination of LGG patient samples. By the transcriptome analysis of nearly 1000 tumors and normal samples from 4 databases, we found that METTL21B mRNA levels are significantly higher in LGG compared with normal tissues and are associated with methylation and CNV of METTL21B. Besides, high METTL21B expression, METL21B hypomethylation and gain/amplification of copy number were found as poor prognostic indicators in TCGA-LGG cohort. Furthermore, the prognostic value of METTL21B expression was also validated in 2 other datasets. Altogether, these findings underlined that METTL21B could be a novel diagnostic and prognostic biomarker in LGG patients.

Function of protein is affected by its methylation modification, which could suggest that methylation of eEF1A by METTL21B have downstream consequences for cellular processes that eEF1A is involved in. In fact, a recent study has unraveled that alterations of METTL21B expression leads to substantial alterations in translation through dynamic regulation of methylation of eEF1A in mammalian cells [23]. Besides its canonical role in translation elongation, eEF1A is also involved in many other cellular activities, such as nuclear export, protein degradation, regulation of the cytoskeleton, apoptosis and so on [37, 38]. eEF1A also plays a vital role in carcinogenesis in multiple cancer types [25]. Additionally, methylation modification of eEF1A at lysine 55 by METTL13 was shown to be 
directly involved in tumorigenesis [26]. However, the mechanism underlying how METTL21B regulate malignant behaviors of LGG by methylation modification of eEF1A remains elusive. By KEGG and GO analysis of DEGs and gene set enrichment analysis, we identified some possible pathways and cellular processed associated with metastasis, tumor immune, angiogenesis and cell proliferation of tumor as above.

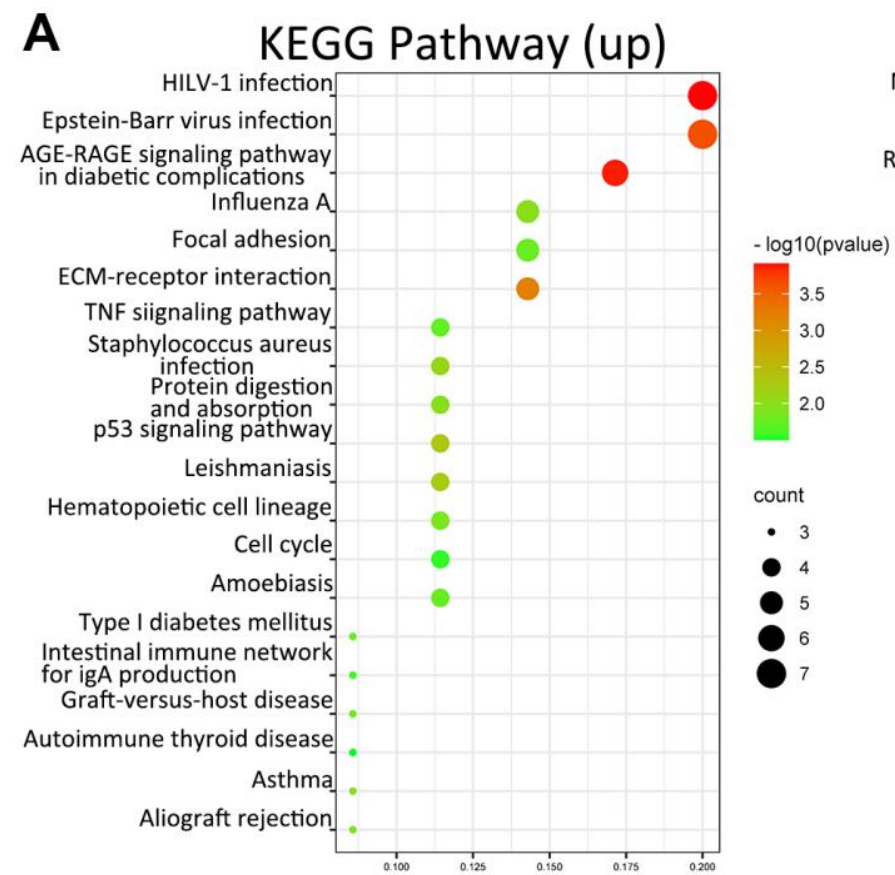

\section{B KEGG Pathway (down)}

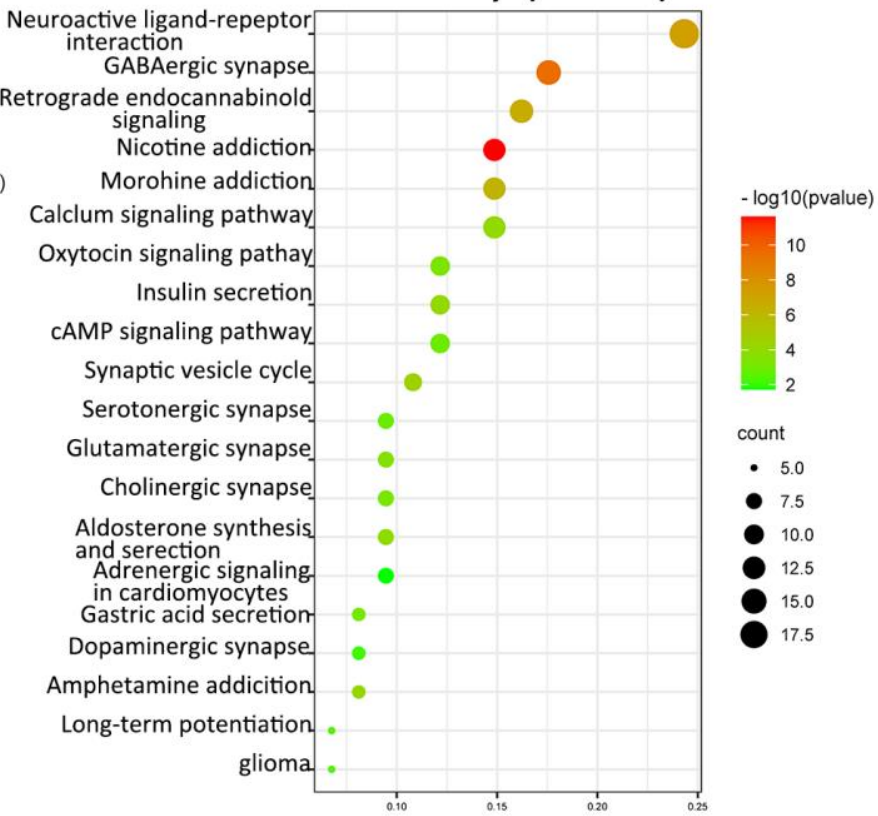

C

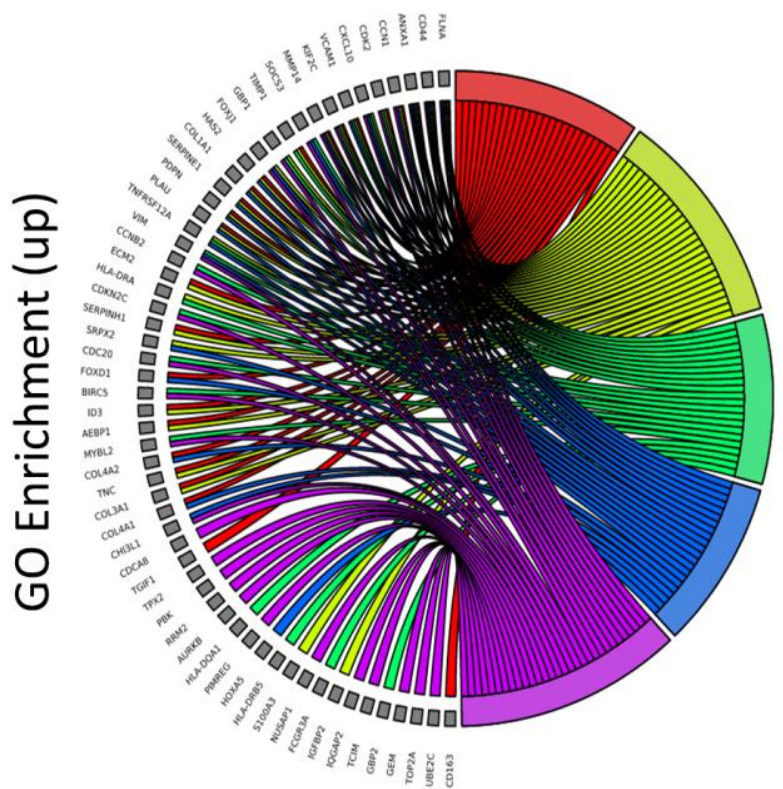

Extracellular matrix organization

$\square$ Regulation of cell adhesion

$\square$ Interferon- $\gamma$-mediated signal

Vasculature development

Cell cycle, mitotic

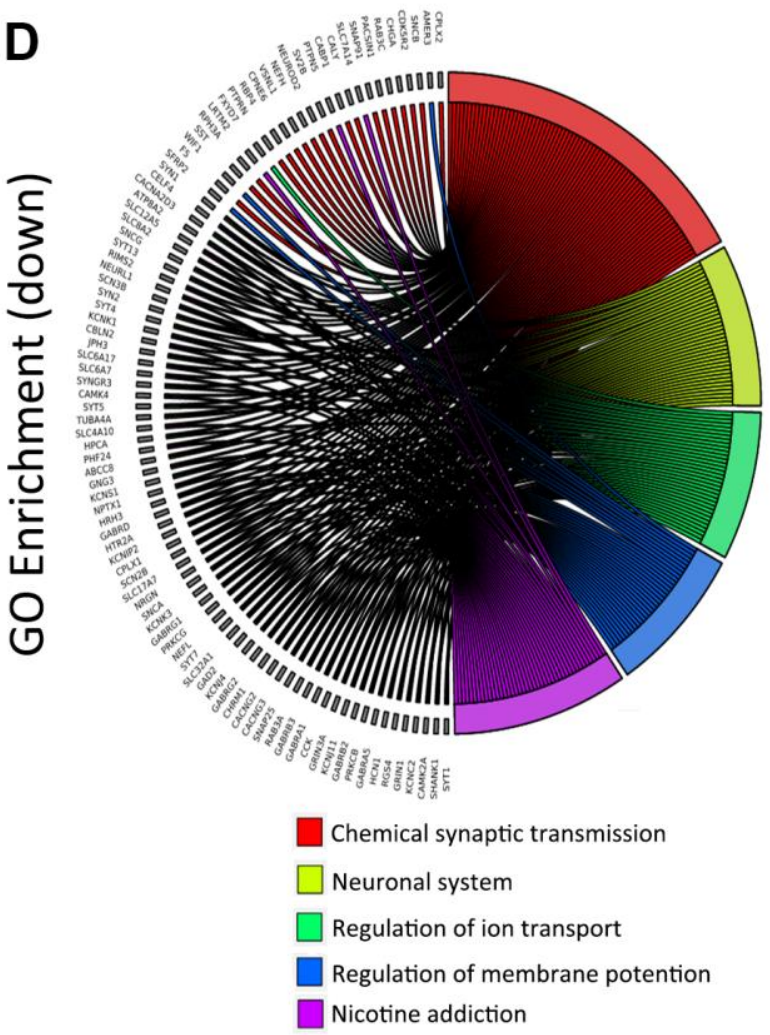

Figure 5. KEGG and GO analysis of DEGs between low and high METTL21B expression groups. KEGG analysis of DEGs (A, B); GO analysis of DEGs (C, D). 
Table 2. 14 Gene sets with normalized enrichment score (NES) $>2.0$ by GSEA.

\begin{tabular}{lcccc}
\hline Gene set name & Size & NES & NOM p-val & FDR q-val \\
\hline HALLMARK_E2F_TARGETS & 195 & 2.799 & 0 & 0 \\
HALLMARK_G2M_CHECKPOINT & 190 & 2.677 & 0 & 0 \\
HALLMARK_INTERFERON_GAMMA_RESPONSE & 198 & 2.560 & 0 & 0 \\
HALLMARK_ALLOGRAFT_REJECTION & 195 & 2.463 & 0 & 0 \\
HALLMARK_EPITHELIAL_MESENCHYMAL_TRANSITION & 197 & 2.442 & 0 & 0 \\
HALLMARK_IL6_JAK_STAT3_SIGNALING & 87 & 2.439 & 0 & 0 \\
HALLMARK_TNFA_SIGNALING_VIA_NFKB & 198 & 2.418 & 0 & 0 \\
HALLMARK_INTERFERON_ALPHA_RESPONSE & 95 & 2.336 & 0 & 0 \\
HALLMARK_ANGIOGENESIS & 36 & 2.291 & 0 & 0 \\
HALLMARK_IL2_STAT5_SIGNALING & 195 & 2.064 & 0 & 0 \\
HALLMARK_INFLAMMATORY_RESPONSE & 198 & 2.063 & 0 & 0 \\
HALLMARK_DNA_REPAIR & 148 & 2.039 & 0 & 0 \\
HALLMARK_APOPTOSIS & 159 & 2.029 & 0 & 0 \\
HALLMARK_MYC_TARGETS_V1 & 194 & 2.013 & 0 & 0 \\
\hline
\end{tabular}

We also discovered that gene targets of MYC family and E2F family were enrichen in high METTL21B group. The E2F-family proteins can induce distinct cell cycle factors to regulate cell proliferation in U343 astrocytoma cells [39]. Besides, MYC also exerts an important effect on the promotion of mitosis in glioma [40]. These results indicate that METTL21B participated in the development of LGG by regulating activity of E2F and MYC transcription factors families.

Accumulating evidence suggests that the tumor immune microenvironment is known to be tightly associated with tumor progression and prognosis of patients [41]. Our results unveiled that METTL21B is significantly positively correlated with infiltration levels of a variety of immune cells, suggesting METTL21B could have a profound influence on the tumor immune microenvironment in LGG. Particularly, macrophage infiltration showed the strongest correlation with METTL21B expression level $(\mathrm{r}=0.415, \mathrm{p}<0.001)$, and was associated with unfavorable prognosis in LGG patients. Furthermore, compared with low METTL21B group, percentage of M2 macrophage is higher in the group with high METTL21B expression. Unlike antitumor function of M1 phenotype, M2 macrophage is deemed immunosuppressive and pro-tumorigenic [42]. Many studies have reported that high levels of TGF- $\beta$, EGF, MMP-2, MMP-9 and IL-10, and low levels of IL12 can be produced by $\mathrm{M} 2$ polarized glioma-associated microglia/macrophages to promotes invasion, proliferation, immune evasion and angiogenesis of glioma [43]. In low-grade glioma, the number of CD68+ (a marker of M2) cells is positively associated with malignancy degree and is inversely related to the recurrence-free survival $[44,45]$. Herein, we speculate that METTL21B may facilitate immune evasion of tumor and worsen prognosis by mediating macrophage polarization from M1 to M2.

A recent study has showed that eEF1A2, a subtype of eEF1A, can regulate expression and release of some cytokines in brain tumors, which implied EEF1A is associated with tumor immune microenvironment [46]. IL-8 was involved in macrophage polarization from M1 to $\mathrm{M} 2$ in glioma, and was founded to be up-regulated in EEF1A2-overexpressed U87-MG cell line [46, 47]. On the other hand, knockdown of EEF1A2 decreased the expression of IL-6 which is considered to play key roles in tumor immune evasion [46, 48, 49]. Thus, METTL21B may regulate tumor immune microenvironment by eEF1A.

As surface membrane receptors of immune cells, immune checkpoints are used by tumors to escape immune surveillance [50]. As mentioned above, patients with high METTL21B expression levels accompanied up-regulation of multiple immune checkpoints, implying a significant role of METTL21B in immune suppression of tumor. On the other hand, patients with high immune checkpoints expression were more likely to benefit from immune checkpoint inhibitors-based immunotherapy [51, 52]. Besides, tumor mutational burden (TMB) has become a potential biomarker and can be utilized to predict immune checkpoint inhibitors efficacy in a host of cancer types [53, 54]. A higher TMB usually results in more neoantigens, thereby increasing immunogenicity of tumor and antitumor activity [53]. Based on this, it is reasonable to assume that patients with high METTL21B level may have better immunotherapeutic response, which is beneficial 
for designing and implementing suitable individualized treatment regimens to improve patients' long-term prognosis.

Except for METTL21B, 4 other methyltransferases have been discovered to be involved in methylation regulation of eEF1A at different sites until now, and eEF1A is also expected to be their only target protein for most of them [36]. Specific methylation sites have distinct functions in modulating eEF1A. For example, upregulation of ribosomal proteins and alternations of some eEF1A-associated cellular processes were
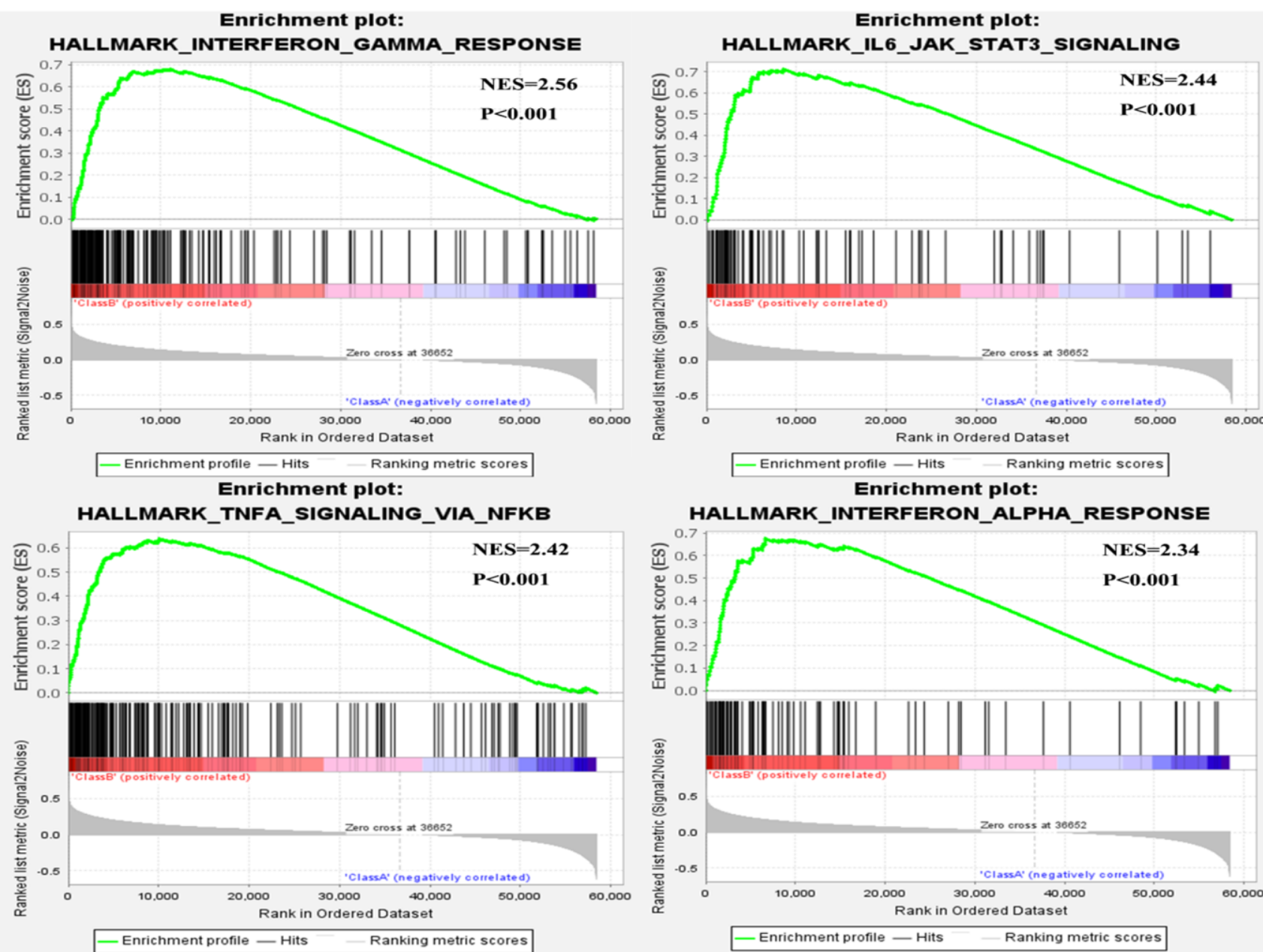

Enrichment plot: HALLMARK_IL2_STAT5_SIGNALING
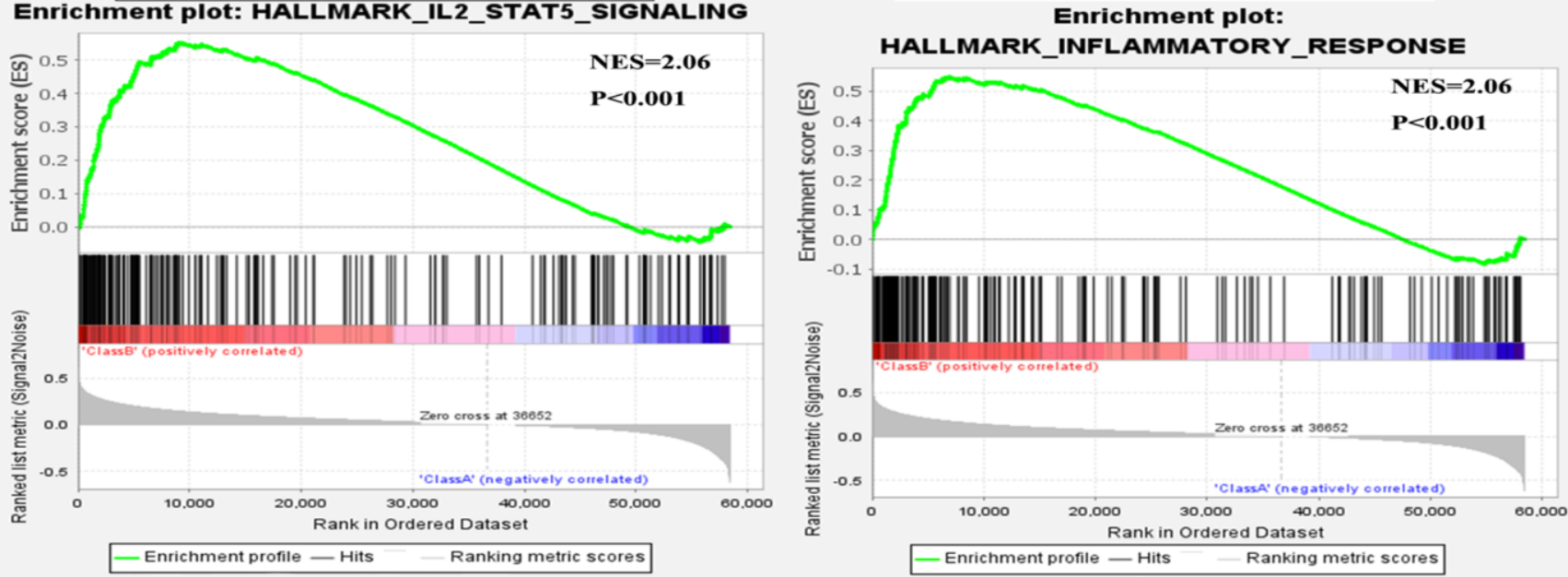

Figure 6. Six immune-related gene sets were enriched by GSEA. 

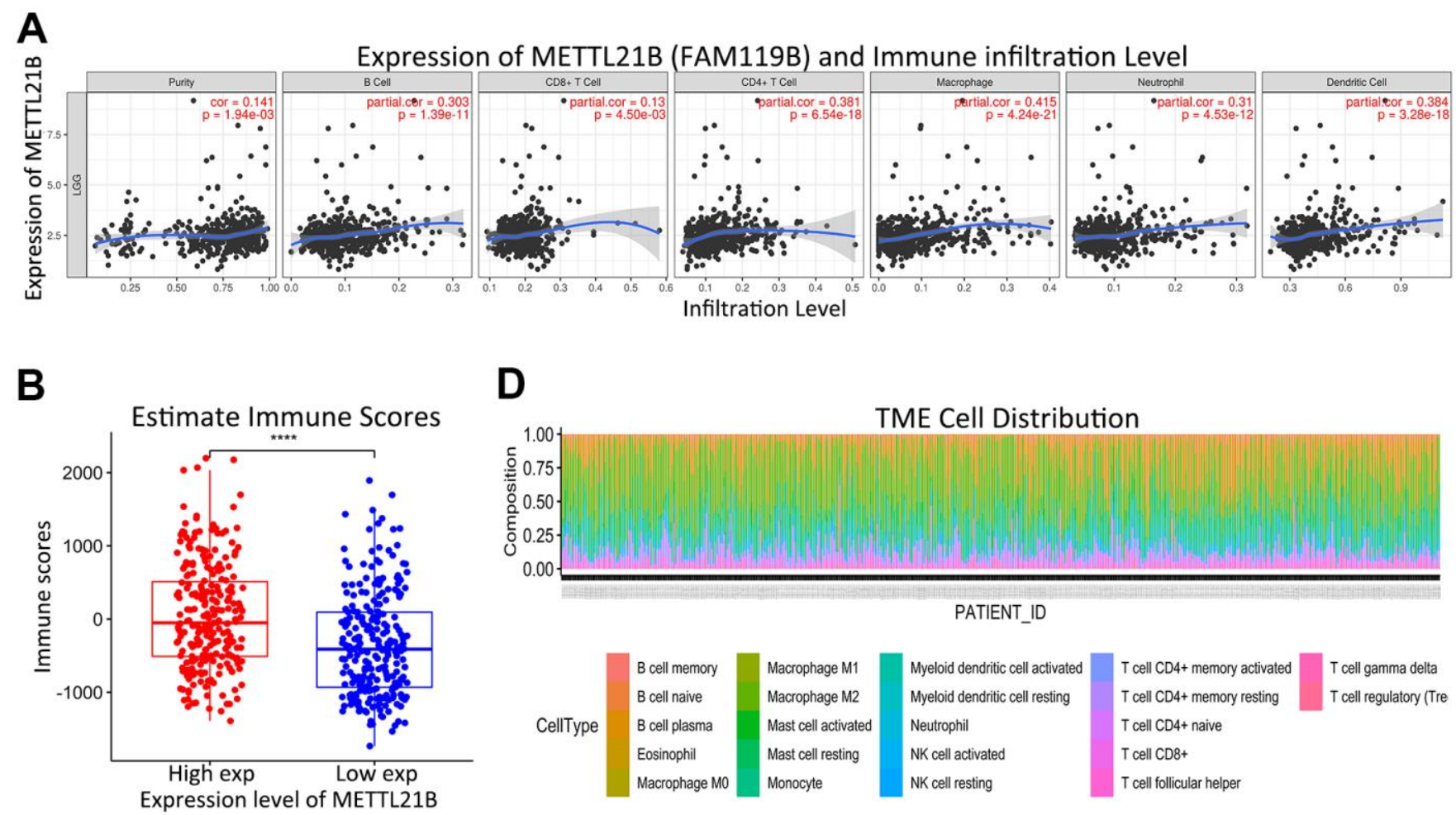

D

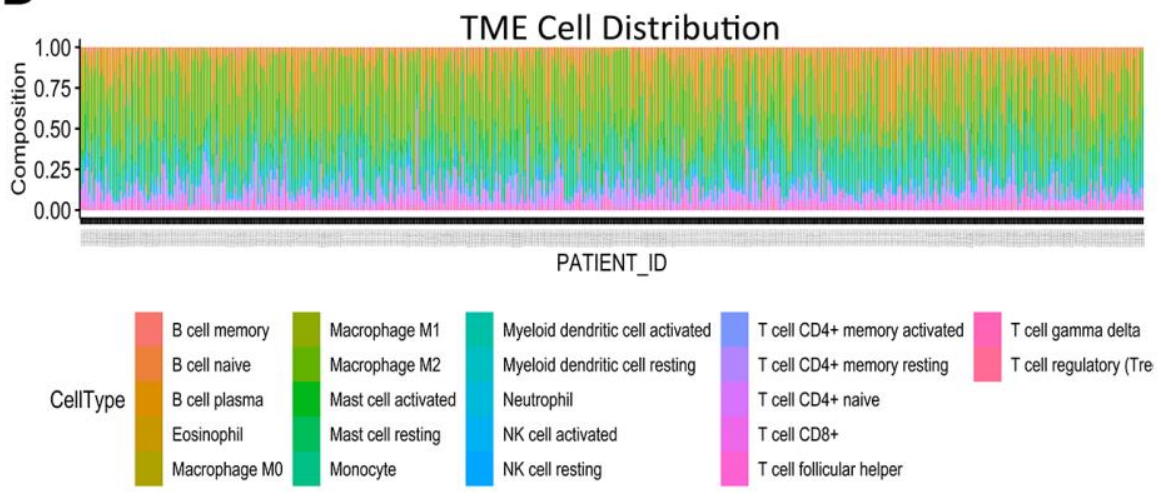

C

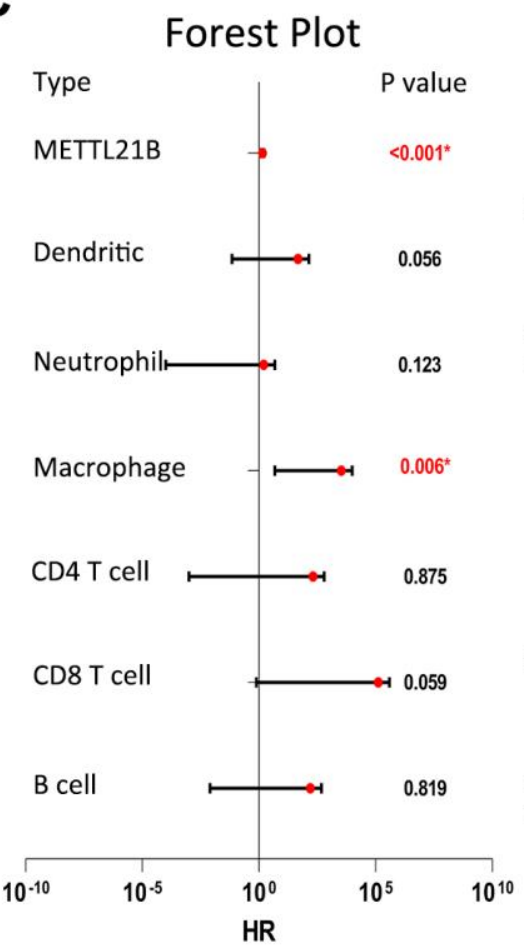

E
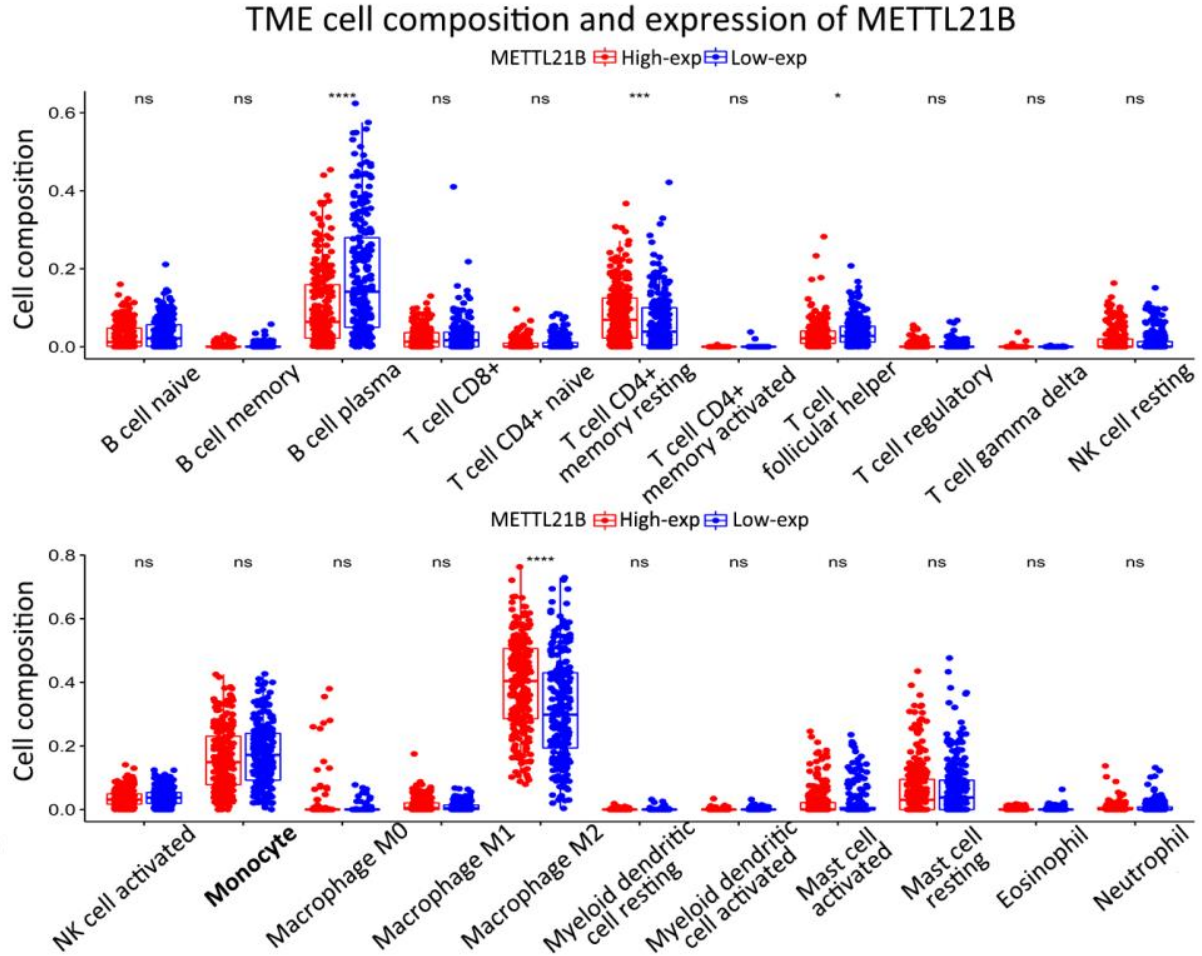

Figure 7. Association between METTL21B and immune infiltration. (A) Infiltration levels of all six immune cell types were significantly positively correlated with expression of METTL21B in LGG by TIMER. (B) Immune scores between low and high METTL21B expression group. (C) Multivariate Cox model for infiltration of six immune cell types and METTL21B expression. (D) Distribution of 22 subtypes of immune cells in all TCGA-LGG samples. (E) Infiltration levels of 22 subtypes of immune cells between low and high METTL21B expression group in LGG. ${ }^{*} \mathrm{P}<0.05 ;{ }^{* * P}<0.01 ;{ }^{* * *} \mathrm{P}<0.001 ;{ }^{* * * * P}<0.0001 ;$ ns: no significance. 
detected in METTL21B-knockout cells compared with EEF1AKMT1-knockout cells by proteomic analysis [24]. In our results, interestingly, EEF1AKMT2 was recognized as a favorable prognostic factor. The finding suggested that functions of eEF1A may be completely opposite when it was methylated at different sites in LGG. Hence, it is tempting to design targeted drugs for the treatment of glioma due to the specificity of METTL21B.
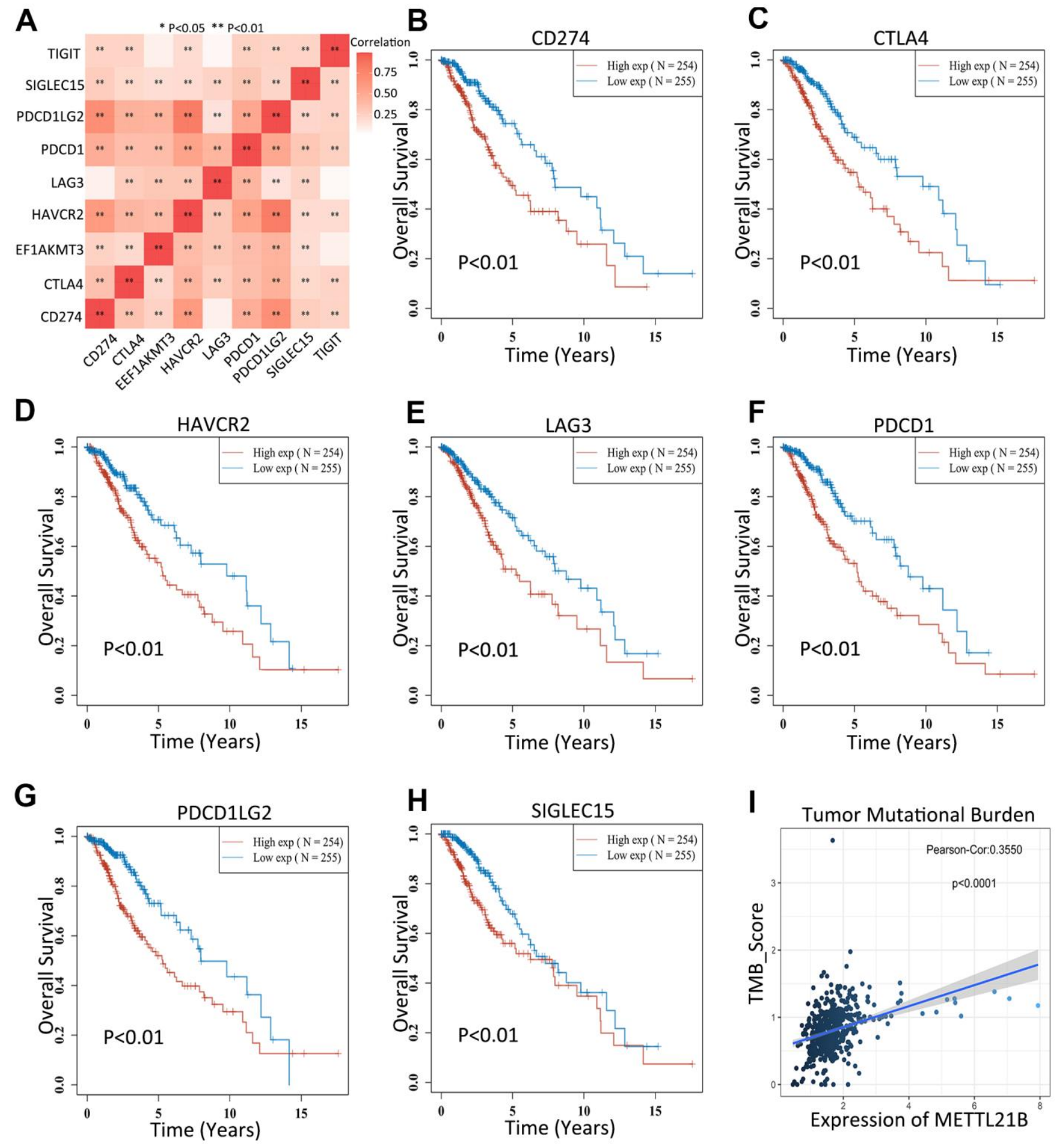

Figure 8. METTL21B is correlated with immune checkpoints and tumor mutation burden. (A) Correlation between expression of 8 immune checkpoints and METTL21B. The effects of CD274 (B), CTLA4 (C), HAVCR2 (D), LAG3 (E), PDCD1 (F), PDCD1LG2 (G) and SIGLEC15 (H) on prognosis of patients with LGG. (I) The correlation between expression of METTL21B and tumor mutation burden in LGG. 
Collectively, the present study confirmed that METTL21B is a promising prognostic biomarker and therapeutic target in LGG for the first time by bioinformatics analysis and experimental validation.
Nevertheless, there are some unavoidable limitations in our study. Firstly, the effect of methylation and CNV of METTL21B on prognosis were unable to be validated in other dataset because of the lack of data. Besides, we

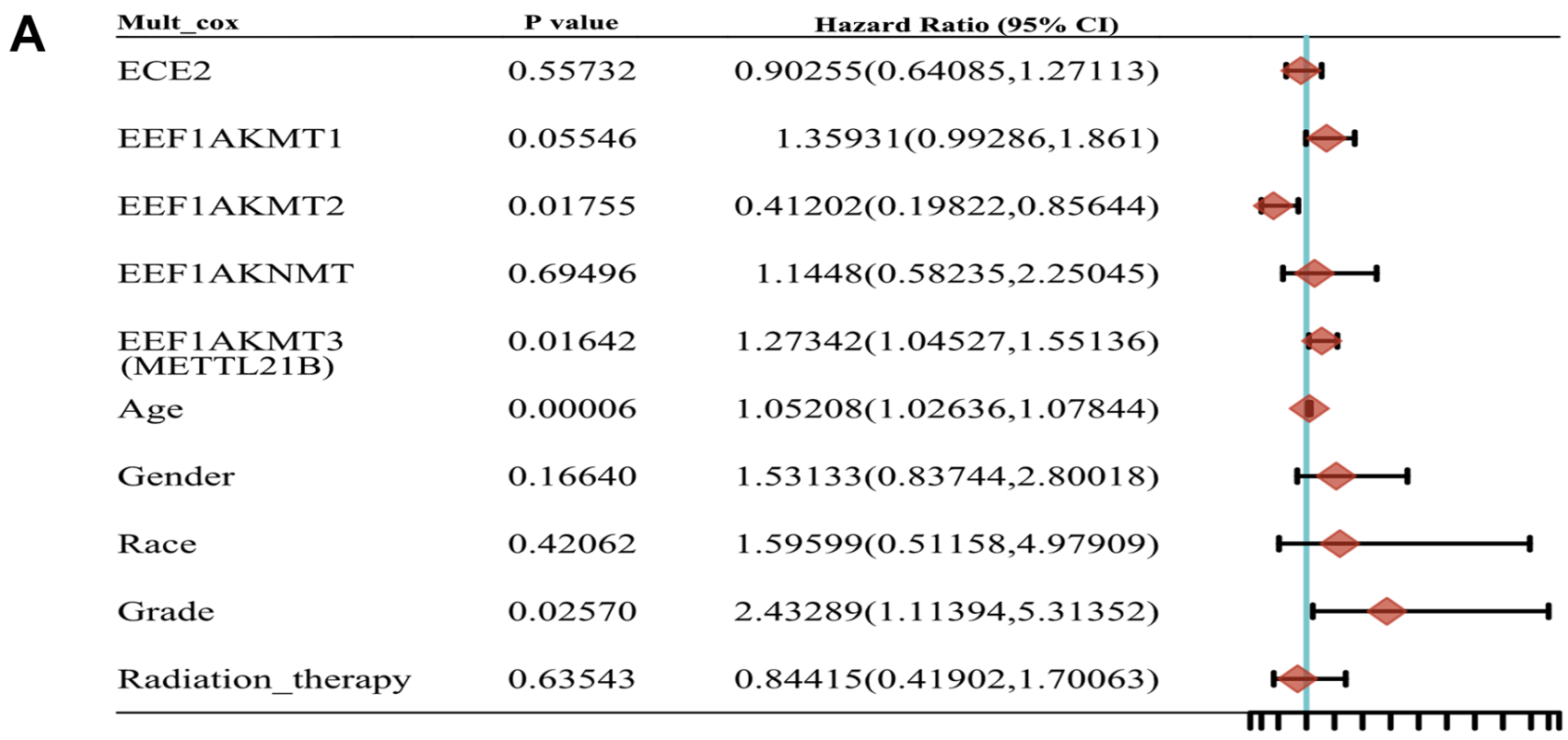

HR: $0.19822 \quad 2 \quad 3 \quad 4 \quad 5$

B

Points

EEF1 AKMT2

EEF 1 AKMT3

(METTL21B)

Age

Grade

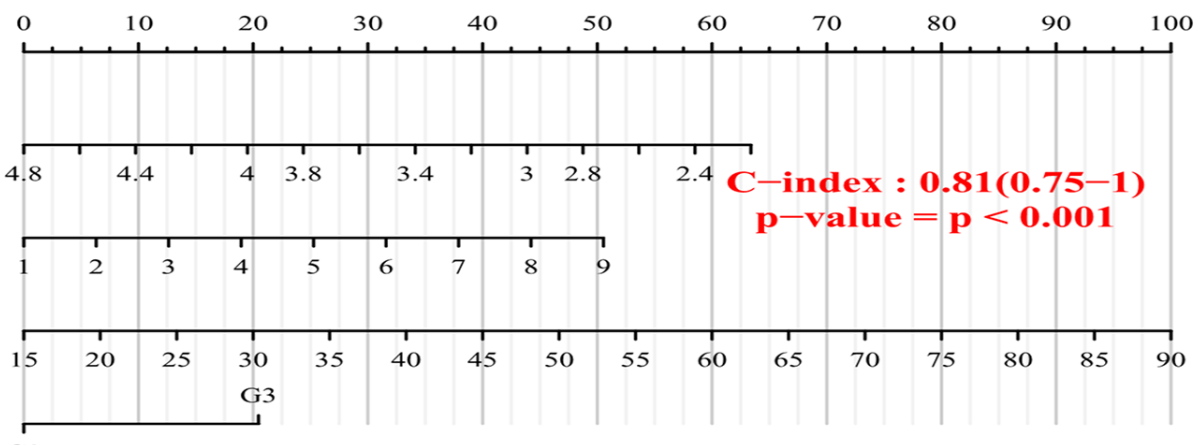

Total Points

Linear Predictor
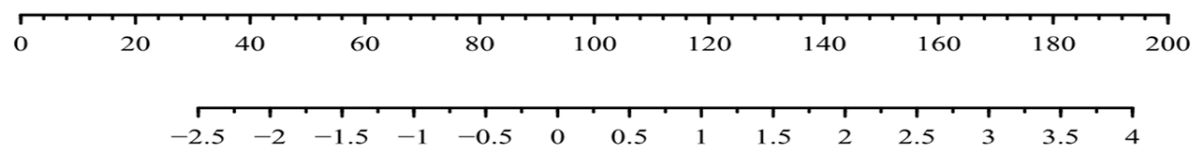

1-year survival Pro

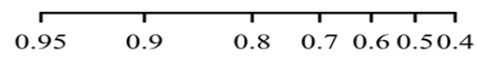

3-year survival Pro

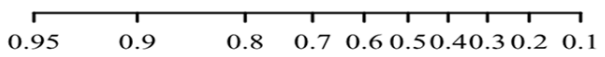

5-year survival Pro

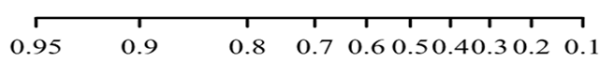

Figure 9. Construction of prognostic model of METTL21B along with other eEF1A associated methyltransferases. (A) Multivariate Cox model for 5 eEF1A associated methyltransferases and clinical features. (B) The nomogram for METTL21B, EEF1AKMT2, age and WHO grade. 
can't obtain the methylation data of eEF1A in LGG from public databases. Thus, the associated between methylation level of eEF1A and METTL21B expression couldn't be analyzed in present study. Finally, further in vitro and in vivo molecular biological experiments are needed be performed to demonstrate concrete mechanisms by which METTL21B regulates malignant behaviors of LGG, especially the correlation with $\mathrm{eEF} 1 \mathrm{~A}$ and tumor immune microenvironment.

\section{AUTHOR CONTRIBUTIONS}

QMW and QW conceived the project; XS, XQL and XCX carried out the database analyses and statistical work; XS wrote the original manuscript; all authors revised the manuscript and approved the final manuscript.

\section{CONFLICTS OF INTEREST}

The authors declare that the research was conducted in the absence of any commercial or financial relationships that could be construed as a potential conflict of interest.

\section{FUNDING}

The experiments in this study were funded by Undergraduate Innovation and Entrepreneurship Training Project (Xin Shu, grant number: 2021).

\section{Editorial note}

${ }^{\&}$ This corresponding author has a verified history of publications using a personal email address for correspondence.

\section{REFERENCES}

1. Binello E, Germano IM. Targeting glioma stem cells: a novel framework for brain tumors. Cancer Sci. 2011; 102:1958-66.

https://doi.org/10.1111/j.1349-7006.2011.02064.x PMID:21848914

2. Louis DN, Perry A, Reifenberger $G$, von Deimling A, Figarella-Branger $D$, Cavenee WK, Ohgaki $\mathrm{H}$, Wiestler OD, Kleihues P, Ellison DW. The 2016 World Health Organization Classification of Tumors of the Central Nervous System: a summary. Acta Neuropathol. 2016; 131:803-20.

https://doi.org/10.1007/s00401-016-1545-1

PMID:27157931

3. Molinaro AM, Taylor JW, Wiencke JK, Wrensch MR. Genetic and molecular epidemiology of adult diffuse glioma. Nat Rev Neurol. 2019; 15:405-17. https://doi.org/10.1038/s41582-019-0220-2 PMID:31227792

4. Keles GE, Lamborn KR, Berger MS. Low-grade hemispheric gliomas in adults: a critical review of extent of resection as a factor influencing outcome. J Neurosurg. 2001; 95:735-45. https://doi.org/10.3171/ins.2001.95.5.0735 PMID:11702861

5. Hayes J, Yu Y, Jalbert LE, Mazor T, Jones LE, Wood MD, Walsh KM, Bengtsson $\mathrm{H}$, Hong $\mathrm{C}$, Oberndorfer $\mathrm{S}$, Roetzer T, Smirnov IV, Clarke JL, et al. Genomic analysis of the origins and evolution of multicentric diffuse lower-grade gliomas. Neuro Oncol. 2018; 20:632-41.

https://doi.org/10.1093/neuonc/nox205 PMID:29077933

6. Cancer Genome Atlas Research Network, Brat DJ, Verhaak RG, Aldape KD, Yung WK, Salama SR, Cooper LA, Rheinbay E, Miller CR, Vitucci M, Morozova O, Robertson AG, Noushmehr $\mathrm{H}$, et al. Comprehensive, Integrative Genomic Analysis of Diffuse Lower-Grade Gliomas. N Engl J Med. 2015; 372:2481-98.

https://doi.org/10.1056/NEJMoa1402121

PMID:26061751

7. Darlix A, Gozé C, Rigau V, Bauchet L, Taillandier L, Duffau $H$. The etiopathogenesis of diffuse low-grade gliomas. Crit Rev Oncol Hematol. 2017; 109:51-62. https://doi.org/10.1016/j.critrevonc.2016.11.014 PMID:28010898

8. Karpel-Massler G, Nguyen TT, Shang E, Siegelin MD. Novel IDH1-Targeted Glioma Therapies. CNS Drugs. 2019; 33:1155-66.

https://doi.org/10.1007/s40263-019-00684-6 PMID:31768950

9. Houillier C, Wang X, Kaloshi G, Mokhtari K, Guillevin R, Laffaire J, Paris S, Boisselier B, Idbaih A, Laigle-Donadey F, Hoang-Xuan K, Sanson M, Delattre JY. IDH1 or IDH2 mutations predict longer survival and response to temozolomide in low-grade gliomas. Neurology. 2010; 75:1560-66.

https://doi.org/10.1212/WNL.0b013e3181f96282 PMID:20975057

10. Mathur R, Zhang $Y$, Grimmer MR, Hong C, Zhang $M$, Bollam S, Petrecca K, Clarke J, Berger MS, Phillips JJ, Oberheim-Bush NA, Molinaro AM, Chang SM, Costello JF. MGMT promoter methylation level in newly diagnosed low-grade glioma is a predictor of hypermutation at recurrence. Neuro Oncol. 2020; 22:1580-90.

https://doi.org/10.1093/neuonc/noaa059 PMID:32166314 
11. Fukuma $R$, Yanagisawa $T$, Kinoshita $M$, Shinozaki $T$, Arita H, Kawaguchi A, Takahashi M, Narita $Y$, Terakawa Y, Tsuyuguchi N, Okita Y, Nonaka M, Moriuchi $S$, et al. Prediction of IDH and TERT promoter mutations in low-grade glioma from magnetic resonance images using a convolutional neural network. Sci Rep. 2019; 9:20311.

https://doi.org/10.1038/s41598-019-56767-3

PMID: $\underline{31889117}$

12. Tan Y, Zhang S, Xiao Q, Wang J, Zhao K, Liu W, Huang K, Tian W, Niu H, Lei T, Shu K. Prognostic significance of ARL9 and its methylation in low-grade glioma. Genomics. 2020; 112:4808-16.

https://doi.org/10.1016/j.ygeno.2020.08.035 PMID:

13. Duan H, Yang Z, Li C, Zhang J, Shen S, Yuan C, Wang Y. Dysregulated Expression and Methylation Analysis Identified TLX1NB as a Novel Recurrence Marker in Low-Grade Gliomas. Int J Genomics. 2020; 2020:5069204.

https://doi.org/10.1155/2020/5069204 PMID:33102572

14. van der Voort SR, Incekara F, Wijnenga MM, Kapas G, Gardeniers M, Schouten JW, Starmans MP, Nandoe Tewarie R, Lycklama GJ, French PJ, Dubbink $\mathrm{HJ}$, van den Bent MJ, Vincent AJ, et al. Predicting the $1 p / 19 q$ Codeletion Status of Presumed LowGrade Glioma with an Externally Validated Machine Learning Algorithm. Clin Cancer Res. 2019; 25:7455-62.

https://doi.org/10.1158/1078-0432.CCR-19-1127 PMID:31548344

15. Moore KE, Gozani O. An unexpected journey: lysine methylation across the proteome. Biochim Biophys Acta. 2014; 1839:1395-403.

https://doi.org/10.1016/j.bbagrm.2014.02.008 PMID:24561874

16. Zhang $X$, Huang $Y$, Shi $X$. Emerging roles of lysine methylation on non-histone proteins. Cell Mol Life Sci. 2015; 72:4257-72. https://doi.org/10.1007/s00018-015-2001-4 PMID:26227335

17. Han D, Huang M, Wang T, Li Z, Chen Y, Liu C, Lei Z, Chu $X$. Lysine methylation of transcription factors in cancer. Cell Death Dis. 2019; 10:290.

https://doi.org/10.1038/s41419-019-1524-2

PMID: 30926778

18. Carlson SM, Gozani O. Nonhistone Lysine Methylation in the Regulation of Cancer Pathways. Cold Spring Harb Perspect Med. 2016; 6:a026435. https://doi.org/10.1101/cshperspect.a026435 PMID:27580749
19. Husmann D, Gozani O. Histone lysine methyltransferases in biology and disease. Nat Struct Mol Biol. 2019; 26:880-89. https://doi.org/10.1038/s41594-019-0298-7 PMID:31582846

20. Martin JL, McMillan FM. SAM (dependent) I AM: the Sadenosylmethionine-dependent methyltransferase fold. Curr Opin Struct Biol. 2002; 12:783-93. https://doi.org/10.1016/s0959-440x(02)00391-3 PMID: 12504684

21. Thalayasingam N, Nair N, Skelton AJ, Massey J, Anderson AE, Clark AD, Diboll J, Lendrem DW, Reynard LN, Cordell HJ, Eyre S, Isaacs JD, Barton A, Pratt AG. CD4+ and B Lymphocyte Expression Quantitative Traits at Rheumatoid Arthritis Risk Loci in Patients With Untreated Early Arthritis: Implications for Causal Gene Identification. Arthritis Rheumatol. 2018; 70:361-70. https://doi.org/10.1002/art.40393 PMID:29193869

22. Ryan KJ, White CC, Patel K, Xu J, Olah M, Replogle JM, Frangieh M, Cimpean M, Winn P, McHenry A, Kaskow BJ, Chan G, Cuerdon N, et al. A human microglia-like cellular model for assessing the effects of neurodegenerative disease gene variants. Sci Transl Med. 2017; 9:eaai7635.

https://doi.org/10.1126/scitranslmed.aai7635 PMID:29263232

23. Malecki J, Aileni VK, Ho AY, Schwarz J, Moen A, Sørensen V, Nilges BS, Jakobsson ME, Leidel SA, Falnes $P \varnothing$. The novel lysine specific methyltransferase METTL21B affects mRNA translation through inducible and dynamic methylation of Lys-165 in human eukaryotic elongation factor 1 alpha (eEF1A). Nucleic Acids Res. 2017; 45:4370-89. https://doi.org/10.1093/nar/gkx002 PMID:28108655

24. Hamey JJ, Wienert B, Quinlan KG, Wilkins MR. METTL21B Is a Novel Human Lysine Methyltransferase of Translation Elongation Factor 1A: Discovery by CRISPR/Cas9 Knockout. Mol Cell Proteomics. 2017; 16:2229-42.

https://doi.org/10.1074/mcp.M116.066308 PMID:28663172

25. Abbas W, Kumar A, Herbein G. The eEF1A Proteins: At the Crossroads of Oncogenesis, Apoptosis, and Viral Infections. Front Oncol. 2015; 5:75. https://doi.org/10.3389/fonc.2015.00075 PMID:25905039

26. Liu S, Hausmann $S$, Carlson SM, Fuentes ME, Francis JW, Pillai R, Lofgren SM, Hulea L, Tandoc K, Lu J, Li A, Nguyen ND, Caporicci M, et al. METTL13 Methylation of eEF1A Increases Translational Output to Promote Tumorigenesis. Cell. 2019; 176:491-504.e21. https://doi.org/10.1016/i.cell.2018.11.038 PMID:30612740 
27. Zhao Z, Zhang $\mathrm{KN}$, Wang $\mathrm{Q}$, Li G, Zeng $\mathrm{F}$, Zhang $\mathrm{Y}$, Wu F, Chai R, Wang Z, Zhang C, Zhang W, Bao Z, Jiang T. Chinese Glioma Genome Atlas (CGGA): A Comprehensive Resource with Functional Genomic Data from Chinese Glioma Patients. Genomics Proteomics Bioinformatics. 2021; S1672-0229: 00045-40.

https://doi.org/10.1016/j.gpb.2020.10.005

PMID:33662628

28. Madhavan S, Zenklusen JC, Kotliarov $Y$, Sahni $H$, Fine HA, Buetow K. Rembrandt: helping personalized medicine become a reality through integrative translational research. Mol Cancer Res. 2009; 7: 157-67.

https://doi.org/10.1158/1541-7786.MCR-08-0435 PMID:19208739

29. Gravendeel LA, Kouwenhoven MC, Gevaert O, de Rooi JJ, Stubbs AP, Duijm JE, Daemen A, Bleeker FE, Bralten LB, Kloosterhof NK, De Moor B, Eilers PH, van der Spek $\mathrm{PJ}$, et al. Intrinsic gene expression profiles of gliomas are a better predictor of survival than histology. Cancer Res. 2009; 69:9065-72.

https://doi.org/10.1158/0008-5472.CAN-09-2307 PMID:19920198

30. Tang Z, Li C, Kang B, Gao G, Li C, Zhang Z. GEPIA: a web server for cancer and normal gene expression profiling and interactive analyses. Nucleic Acids Res. 2017; 45:W98-102.

https://doi.org/10.1093/nar/gkx247 PMID:28407145

31. Gao J, Aksoy BA, Dogrusoz U, Dresdner G, Gross B, Sumer SO, Sun $Y$, Jacobsen A, Sinha R, Larsson E, Cerami E, Sander C, Schultz N. Integrative analysis of complex cancer genomics and clinical profiles using the cBioPortal. Sci Signal. 2013; 6:pl1.

https://doi.org/10.1126/scisignal.2004088

PMID:23550210

32. Wang J, Voellger B, Benzel J, Schlomann U, Nimsky C, Bartsch JW, Carl B. Metalloproteinases ADAM12 and MMP-14 are associated with cavernous sinus invasion in pituitary adenomas. Int J Cancer. 2016; 139:1327-39.

https://doi.org/10.1002/ijc.30173

PMID:27144841

33. Li T, Fan J, Wang B, Traugh N, Chen Q, Liu JS, Li B, Liu XS. TIMER: A Web Server for Comprehensive Analysis of Tumor-Infiltrating Immune Cells. Cancer Res. 2017; 77:e108-10.

https://doi.org/10.1158/0008-5472.CAN-17-0307 PMID:29092952

34. Sun J, Lu Q, Sanmamed MF, Wang J. Siglec-15 as an Emerging Target for Next-generation Cancer Immunotherapy. Clin Cancer Res. 2021; 27:680-88. https://doi.org/10.1158/1078-0432.CCR-19-2925
PMID:32958700

35. Qin S, Xu L, Yi M, Yu S, Wu K, Luo S. Novel immune checkpoint targets: moving beyond PD-1 and CTLA-4. Mol Cancer. 2019; 18:155. https://doi.org/10.1186/s12943-019-1091-2 PMID:31690319

36. Hamey JJ, Wilkins MR. Methylation of Elongation Factor 1A: Where, Who, and Why? Trends Biochem Sci. 2018; 43:211-23. https://doi.org/10.1016/j.tibs.2018.01.004 PMID:29398204

37. Mateyak MK, Kinzy TG. eEF1A: thinking outside the ribosome. J Biol Chem. 2010; 285:21209-13. https://doi.org/10.1074/jbc.R110.113795 PMID:20444696

38. Sasikumar AN, Perez WB, Kinzy TG. The many roles of the eukaryotic elongation factor 1 complex. Wiley Interdiscip Rev RNA. 2012; 3:543-55.

https://doi.org/10.1002/wrna.1118 PMID:22555874

39. Dirks PB, Rutka JT, Hubbard SL, Mondal S, Hamel PA. The E2F-family proteins induce distinct cell cycle regulatory factors in p16-arrested, U343 astrocytoma cells. Oncogene. 1998; 17:867-76.

https://doi.org/10.1038/sj.onc.1202008 PMID: 9780003

40. Annibali D, Whitfield JR, Favuzzi E, Jauset T, Serrano E, Cuartas I, Redondo-Campos S, Folch G, Gonzàlez-Juncà A, Sodir NM, Massó-Vallés D, Beaulieu ME, Swigart LB, et al. Myc inhibition is effective against glioma and reveals a role for Myc in proficient mitosis. Nat Commun. 2014; 5:4632.

https://doi.org/10.1038/ncomms5632 PMID:25130259

41. Hinshaw DC, Shevde LA. The Tumor Microenvironment Innately Modulates Cancer Progression. Cancer Res. 2019; 79:4557-66. https://doi.org/10.1158/0008-5472.CAN-18-3962 PMID:31350295

42. Xia $Y$, Rao L, Yao H, Wang $Z$, Ning $P$, Chen $X$. Engineering Macrophages for Cancer Immunotherapy and Drug Delivery. Adv Mater. 2020; 32:e2002054. https://doi.org/10.1002/adma.202002054 PMID:32856350

43. Gieryng A, Pszczolkowska D, Walentynowicz KA, Rajan WD, Kaminska B. Immune microenvironment of gliomas. Lab Invest. 2017; 97:498-518. https://doi.org/10.1038/labinvest.2017.19 PMID:28287634

44. Komohara Y, Ohnishi K, Kuratsu J, Takeya M. Possible involvement of the $\mathrm{M} 2$ anti-inflammatory macrophage phenotype in growth of human gliomas. J Pathol. 2008; 216:15-24. 
https://doi.org/10.1002/path.2370

PMID:18553315

45. Alford R, Gargan L, Bowers DC, Klesse LJ, Weprin B, Koral K. Postoperative surveillance of pediatric cerebellar pilocytic astrocytoma. J Neurooncol. 2016; 130:149-54.

https://doi.org/10.1007/s11060-016-2222-x PMID:27502785

46. Hassan MK, Kumar D, Patel SA, Pattanaik N, Mohapatra N, Dixit M. Expression pattern of EEF1A2 in brain tumors: Histological analysis and functional role as a promoter of EMT. Life Sci. 2020; 246:117399. https://doi.org/10.1016/j.Ifs.2020.117399 PMID: 32032648

47. Xu Y, Liao C, Liu R, Liu J, Chen Z, Zhao H, Li Z, Chen L, Wu C, Tan H, Liu W, Li W. IRGM promotes glioma M2 macrophage polarization through p62/TRAF6/NF-kB pathway mediated IL-8 production. Cell Biol Int. 2019; 43:125-35.

https://doi.org/10.1002/cbin.11061

PMID:30288851

48. Weng YS, Tseng HY, Chen YA, Shen PC, Al Haq AT, Chen LM, Tung YC, Hsu HL. MCT-1/miR-34a/IL-6/IL-6R signaling axis promotes EMT progression, cancer stemness and M2 macrophage polarization in triplenegative breast cancer. Mol Cancer. 2019; 18:42. https://doi.org/10.1186/s12943-019-0988-0 PMID:30885232

49. Yin Z, Ma T, Lin Y, Lu X, Zhang C, Chen S, Jian Z. IL6/STAT3 pathway intermediates M1/M2 macrophage polarization during the development of hepatocellular carcinoma. J Cell Biochem. 2018; 119:9419-32.

https://doi.org/10.1002/jcb.27259

PMID:30015355

50. Pardoll DM. The blockade of immune checkpoints in cancer immunotherapy. Nat Rev Cancer. 2012; $12: 252-64$. https://doi.org/10.1038/nrc3239

PMID:22437870

51. Brody $R$, Zhang $Y$, Ballas $M$, Siddiqui $M K$, Gupta $P$, Barker C, Midha A, Walker J. PD-L1 expression In advanced NSCLC: Insights into risk stratification and treatment selection from a systematic literature review. Lung Cancer. 2017; 112:200-15. https://doi.org/10.1016/i.lungcan.2017.08.005 PMID:29191596

52. Aguilar EJ, Ricciuti B, Gainor JF, Kehl KL, Kravets $S$, Dahlberg S, Nishino M, Sholl LM, Adeni A, Subegdjo S, Khosrowjerdi S, Peterson RM, Digumarthy $S$, et al. Outcomes to first-line pembrolizumab in patients with non-small-cell lung cancer and very high PD-L1 expression. Ann Oncol. 2019; 30:1653-59. https://doi.org/10.1093/annonc/mdz288 PMID:31435660

53. Chan TA, Yarchoan M, Jaffee E, Swanton C, Quezada SA, Stenzinger A, Peters S. Development of tumor mutation burden as an immunotherapy biomarker: utility for the oncology clinic. Ann Oncol. 2019; 30:44-56.

https://doi.org/10.1093/annonc/mdy495 PMID:30395155

54. Bouffet E, Larouche V, Campbell BB, Merico D, de Borja R, Aronson M, Durno C, Krueger J, Cabric V, Ramaswamy V, Zhukova N, Mason G, Farah R, et al. Immune Checkpoint Inhibition for Hypermutant Glioblastoma Multiforme Resulting From Germline Biallelic Mismatch Repair Deficiency. J Clin Oncol. 2016; 34:2206-11. https://doi.org/10.1200/JCO.2016.66.6552 PMID:27001570 


\section{SUPPLEMENTARY MATERIALS}

\section{Supplementary Figures}

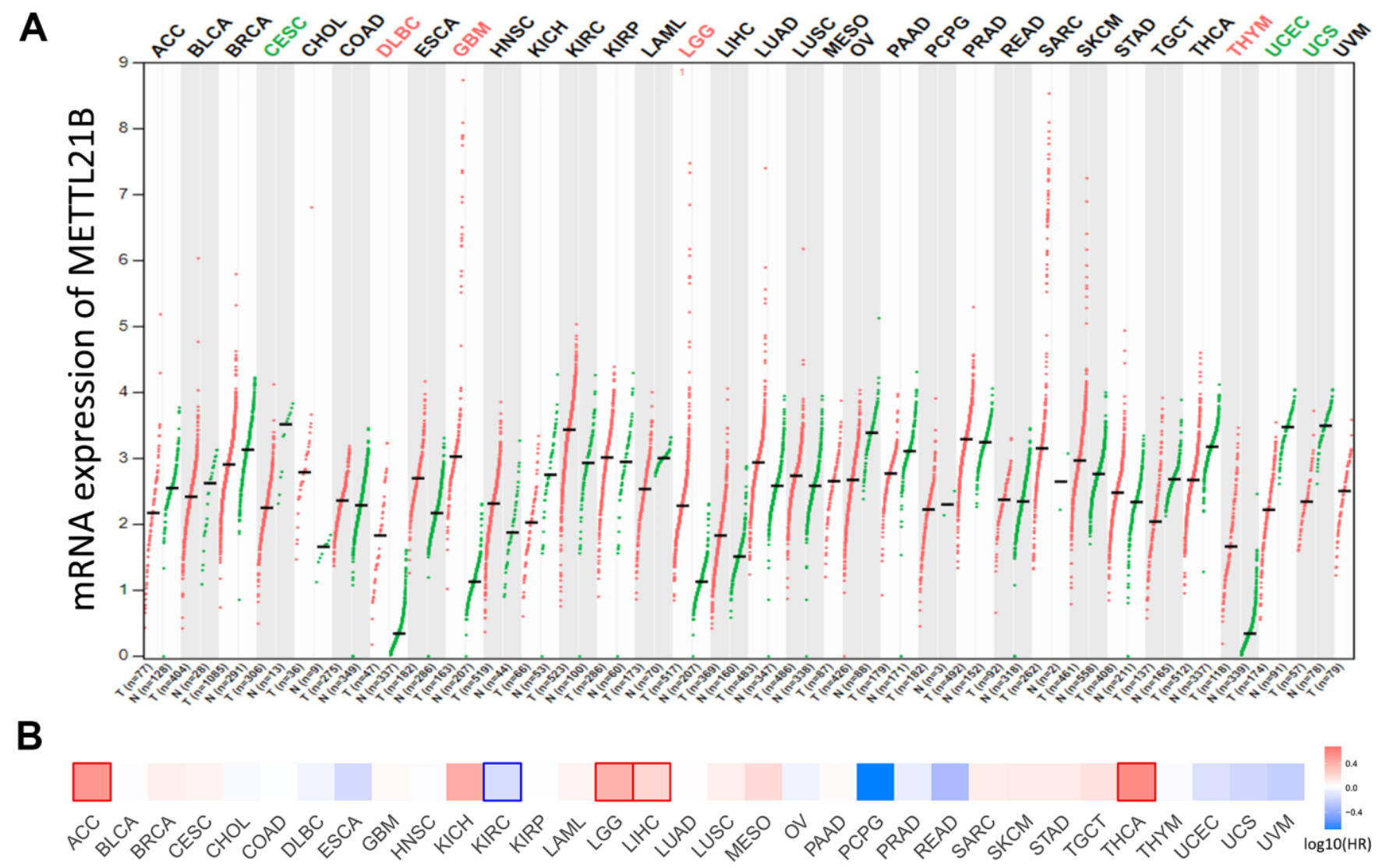

Supplementary Figure 1. Pan-cancer analysis of METTL21B expression among 33 types of cancers from TCGA. (A) The difference in expression of METTL21B between tumor tissues and normal tissues of 33 types of human cancers from TCGA and GTEx database. Red/Green fonts indicate statistically significant differences. (B) Survival heat map of 33 cancer types in TCGA. Red/Blue border lines indicate statistically significant differences. 
A

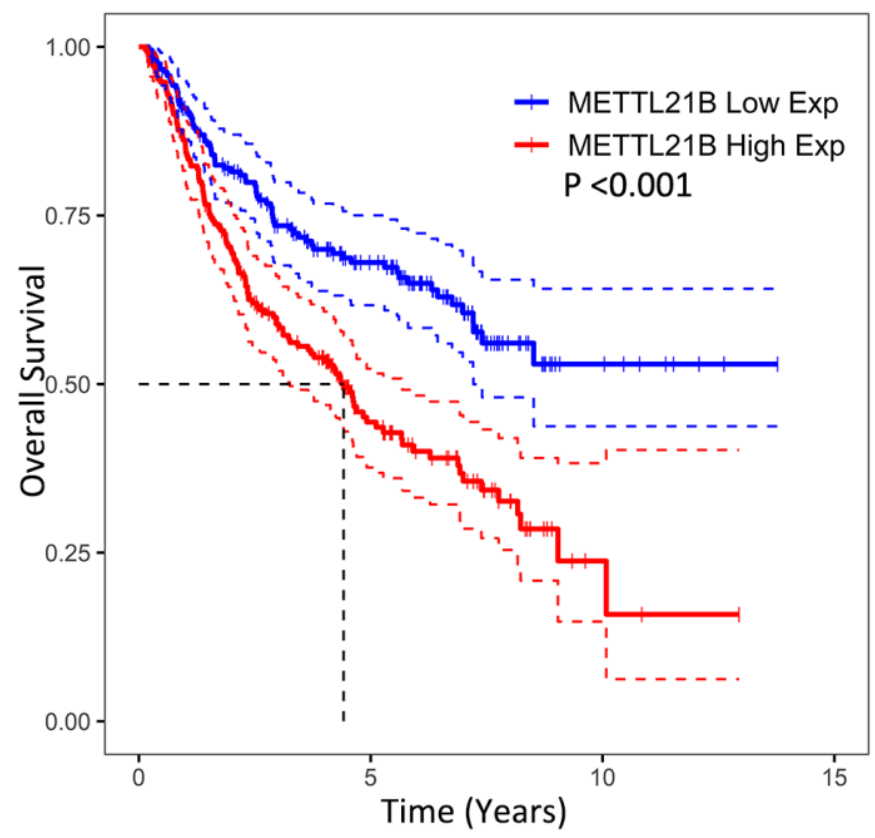

B

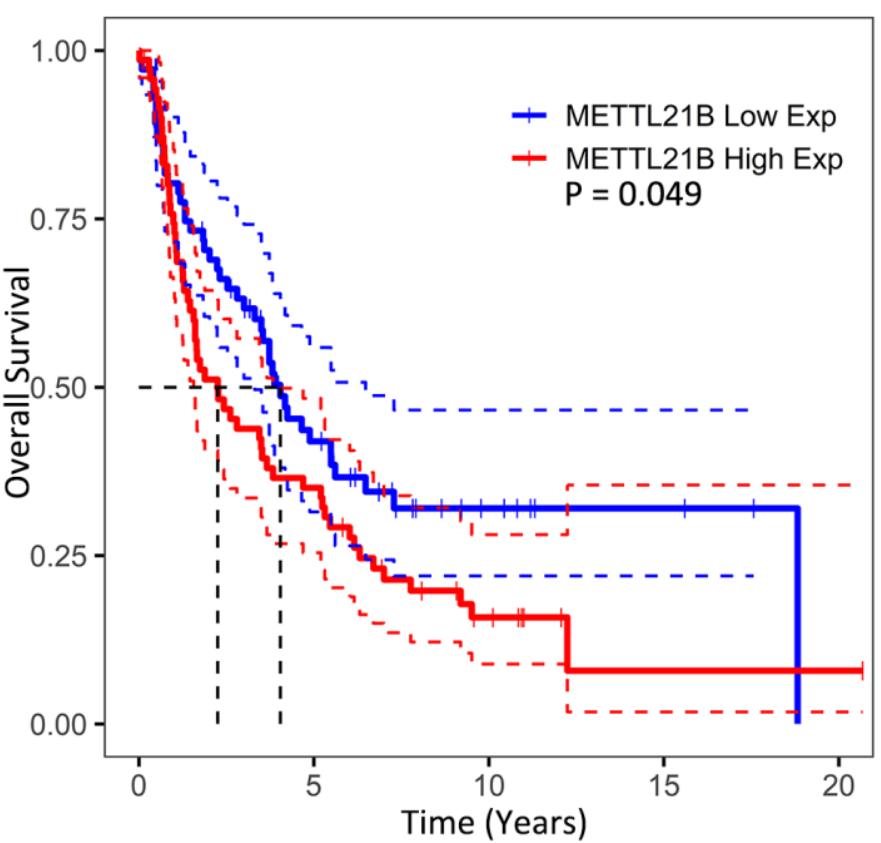

Supplementary Figure 2. The effect of METTL21B expression on prognosis of patients with LGG in CGGA database (A) and Rembrandt database (B).

A

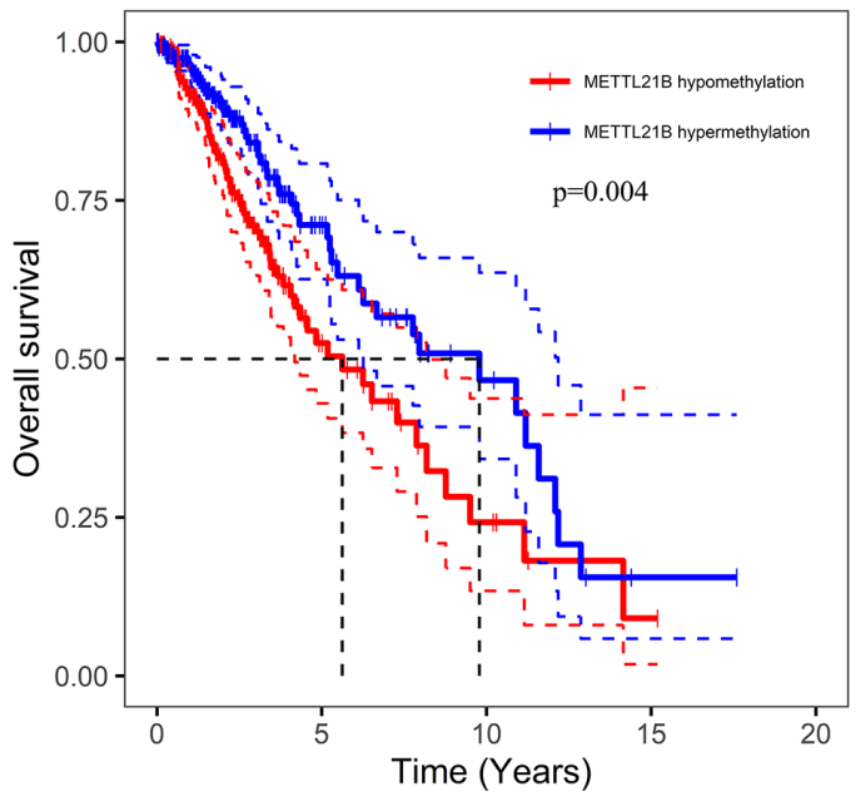

B

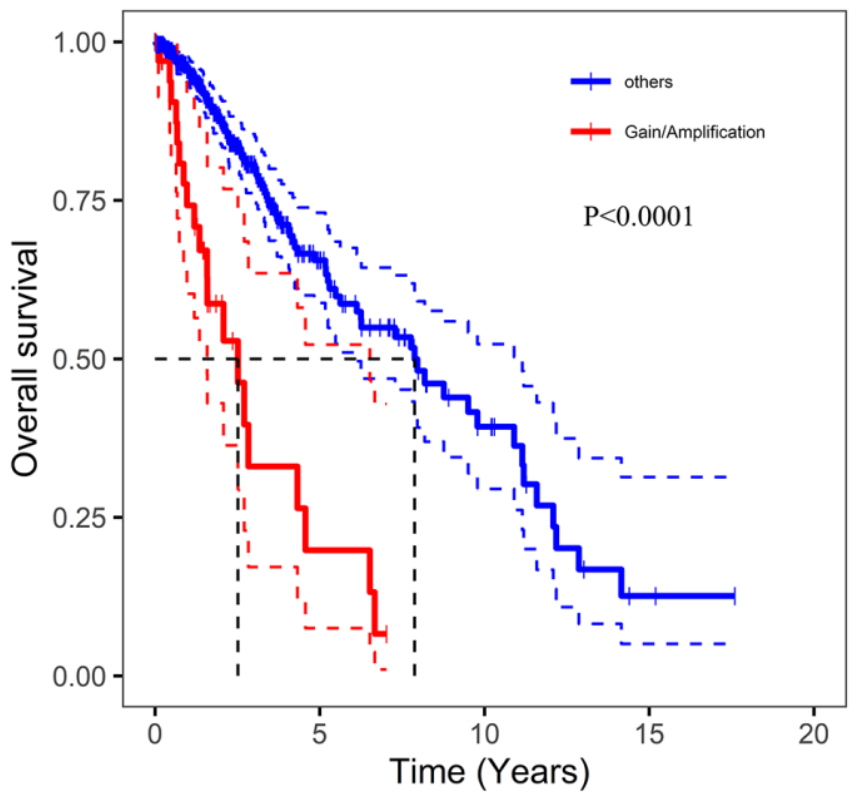

Supplementary Figure 3. The effect of METTL21B methylation (A) and CNV (B) on prognosis of patients with LGG. 
A

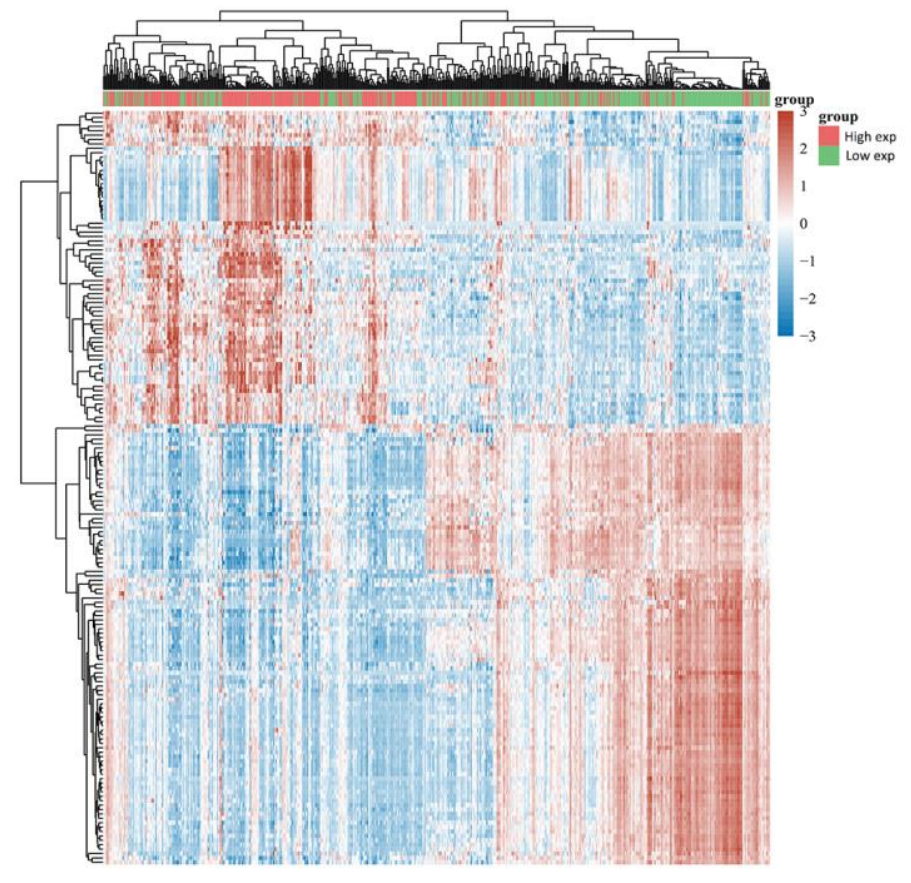

B

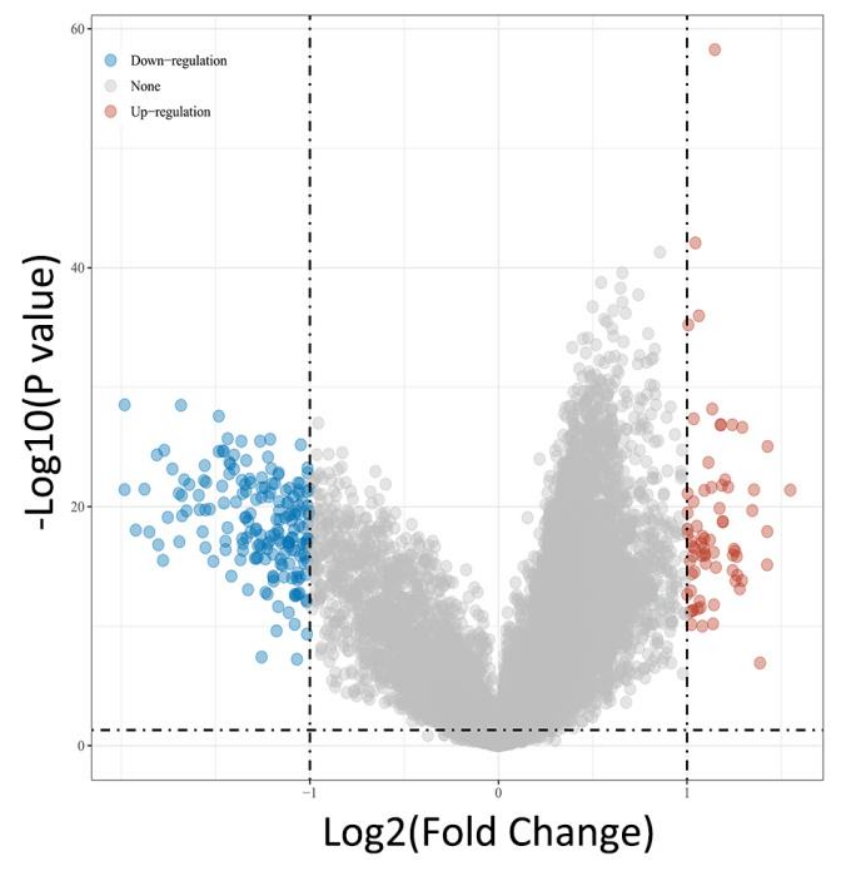

Supplementary Figure 4. Heatmap (A) and volcano plot (B) for DEGs.

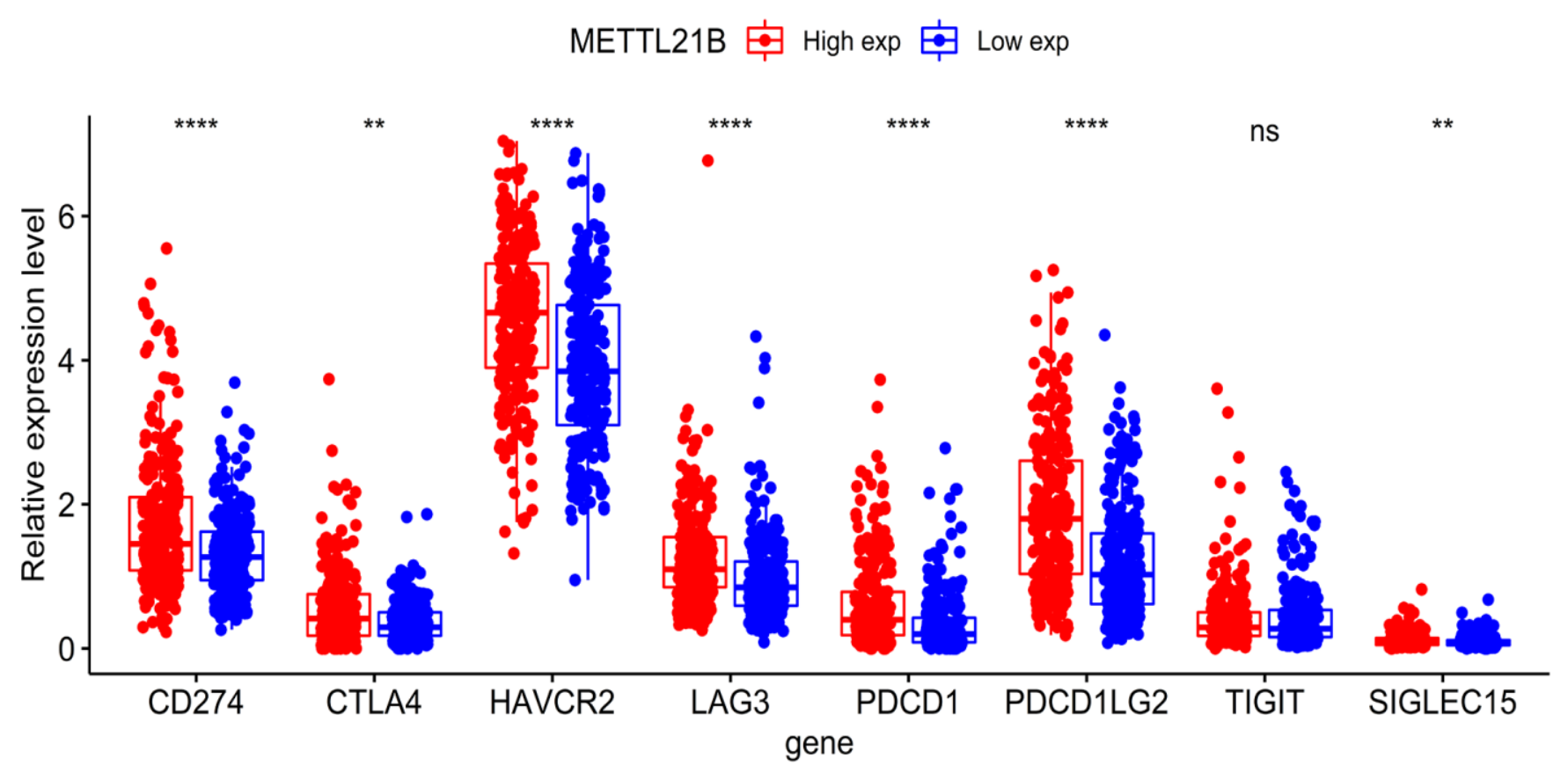

Supplementary Figure 5. The expression differences of 8 immune checkpoints between low and high METTL21B expression group. ${ }^{* *} \mathrm{P}<0.01 ;{ }^{* * * * P}<0.0001$; ns: no significance. 


\section{Supplementary Table}

Supplementary Table 1. The primers used for qPCR in this study.

\begin{tabular}{lcc}
\hline Gene & Forward primer & Reverse primer \\
\hline h- METTL21B & GTGGATTTCCGAGGCAAGAAGG & CAGGTCAGTGATGGTAACATCCC \\
h-GAPDH & AGGGGCCATCCACAGTCTTC & AGAAGGCTGGGGCTCATTTG \\
\hline
\end{tabular}

